\title{
Tunable Metasurfaces: A Polarization Rotator Design
}

\author{
Zhanni Wu, ${ }^{1}$ Younes Ra'di, ${ }^{1,2,3}$ and Anthony Grbic ${ }^{1}$ \\ ${ }^{1}$ Department of Electrical Engineering and Computer Science, \\ University of Michigan, Ann Arbor, Michigan 48109-2122, USA \\ ${ }^{2}$ Department of Electrical and Computer Engineering, The University of Texas, Austin, Texas 78712, USA \\ ${ }^{3}$ Photonics Initiative, Advanced Science Research Center, City University of New York, \\ New York City, New York 10031, USA
}

(Received 21 March 2018; revised manuscript received 27 November 2018; published 22 February 2019)

\begin{abstract}
Metasurfaces have enabled unprecedented control over electromagnetic waves, providing new opportunities in areas such as wireless communications, energy harvesting, imaging, and cloaking. Despite significant progress in this area, most metasurface designs provide static functionalities. Here, we theoretically propose and experimentally validate a transparent metasurface with dynamically tunable properties. Notably, we present an electronically tunable metasurface that is capable of rotating the polarization of an arbitrarily polarized incident wave, without changing its axial ratio. The designed polarization rotator consists of a tunable birefringent structure sandwiched between two $\pm 45^{\circ}$ rotated metasurface-based quarter-wave plates. The birefringent structure is a bandpass metasurface which relies on integrated varactor diodes for tunability. Through a simple biasing mechanism, different voltages are applied to the diodes to rotate the polarization of a transmitted wave. The proposed idea may find application in various areas where dynamic polarization control is required.
\end{abstract}

DOI: 10.1103/PhysRevX.9.011036

Subject Areas: Metamaterials

\section{INTRODUCTION}

In recent years, a great deal of attention has been devoted to tailoring electromagnetic wave fronts using metasurfaces. Metasurfaces are surfaces textured at a subwavelength scale to achieve tailored electromagnetic responses. The reduced dimensionality of metasurfaces, compared to their three-dimensional counterparts, metamaterials, makes them less lossy and easily integrable.

To date, metasurfaces with various functionalities have been introduced and practically realized [1-6]. These electrically thin layers have found applications in areas such as microwave and millimeter-wave antennas, flat optical components, energy harvesting, and terahertz devices. However, most reported metasurfaces have static functionalities that depend on their fixed geometrical parameters. In other words, their electric, magnetic, and magnetoelectric responses are fixed by design, and cannot be changed after fabrication.

From a practical perspective, dynamic control over a metasurface's response is highly desired. Tunable metasurfaces can offer a multifunctional platform that dynamically manipulates electromagnetic wave fronts. There have been various developments in the emerging area of tunable

Published by the American Physical Society under the terms of the Creative Commons Attribution 4.0 International license. Further distribution of this work must maintain attribution to the author(s) and the published article's title, journal citation, and DOI. metasurfaces utilizing liquid crystals $[7,8]$, phase transition materials [9], nonlinear optical materials [10,11], voltagecontrolled doping [12], and mechanical tuning [13,14]. Recently, dynamically tuning the scattering characteristics of metasurfaces has been the subject of several studies which have resulted in metasurfaces for tunable beam steering, absorption, and change of polarization state [15-22]. However, most of the reported designs provide limited tunability, or they achieve the tunability at the cost of reduced operational bandwidth and overall efficiency. Furthermore, most of the reported tunable metasurfaces provide their tunable functionalities in reflection [15-19]. Achieving tunable scattering characteristics in transmission is more challenging since in ideal, tunable transmissive metasurfaces, reflection should be zero over the entire range of tunability. Although a few attempts have been made to design tunable transmissive metasurfaces $[13,14,20-22]$, the reported structures provide limited tunability and performance. Another challenging issue common to most of these tunable metasurfaces is the limitations imposed by biasing networks on their performance and realization. Designing biasing networks that do not interfere with the wave-front-shaping functionalities of these tunable metasurfaces is of significant importance. In tunable reflective metasurfaces, the biasing network is typically located behind a ground plane to minimize its interaction with incoming wave fronts. However, in tunable transmissive metasurfaces, there is no ground plane to isolate the biasing network from the rest of the structure. 
Finally, tunable metasurfaces require controllable elements. For example, at microwave frequencies, tunable metasurface designs have relied heavily on varactor diodes [23-25], which introduce added losses.

Here, we develop a compact, configurable metasurface, functioning as a transmissive electronically tunable polarization rotator, with a relatively high efficiency and large tunability range. In the reported design, not only does the biasing network not disturb the microwave performance of the metasurface, but it serves as an inductive element essential to the metasurface's functionality. Although, a metasurface is designed that provides tunable polarization control in transmission, the design principles outlined can enable full control over the transmitted wave, both tunable polarization and beam steering. In other words, with a more sophisticated biasing network, the designed metasurface could provide dynamic control over the direction and polarization of the transmitted wave.

Polarization control is one of the most fundamental functionalities that can be offered by metasurfaces. The proposed structure is capable of dynamically varying the tilt angle of the transmitted linearly or, in general, elliptically polarized waves by an arbitrary angle (rotation angle $\theta_{r}$ ) through a simple biasing system. In addition to rotating the polarization of the input wave, the proposed structure also serves as a tunable phase shifter for circularly polarized waves. Compared to static designs [26-29], the reported design provides a high degree of freedom, while maintaining a reasonable efficiency and bandwidth performance, given the number of impedance sheets and active elements integrated. The tunability offered by the design may provide new opportunities in application areas such as radar detection, polarimetric imaging, and millimeter-wave communication system.

\section{VARIABLE POLARIZATION ROTATOR: JONES MATRIX ANALYSIS}

The proposed polarization rotator consists of a tunable birefringent structure (BFS) placed between two quarterwave plates (QWPs) [29]. The geometry of the system is shown in Fig. 1. Let us begin by considering the Jones matrices for the QWPs and tunable BFS separately. The QWPs are rotated $+45^{\circ}$ and $-45^{\circ}$ with respect to the optical axis of the BFS. For an ideal QWP, the phase delays for two orthogonal polarizations differ by $90^{\circ}$, and the transmittance for each polarization is 1 . Thus, the Jones matrix of a generic QWP, aligned with the $x-y$ coordinate axis, is

$$
\mathbf{Q}=e^{j \alpha}\left[\begin{array}{cc}
1 & 0 \\
0 & e^{j \pi / 2}
\end{array}\right],
$$

where $\alpha$ is a phase delay common to both optical axes of the QWP. If an optical device is rotated counterclockwise by an angle $\theta$ about the origin, the Jones matrix for the rotated device becomes

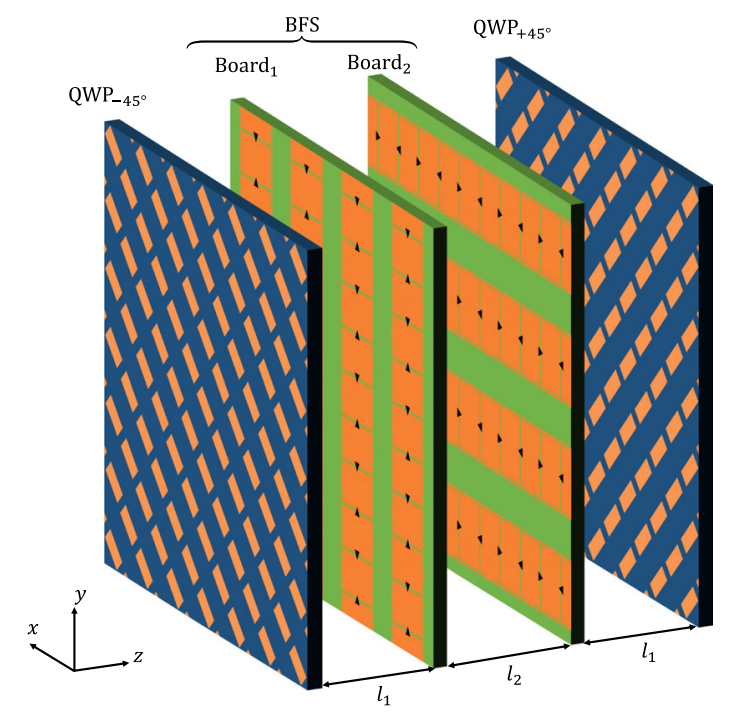

FIG. 1. Topology of the polarization rotator. The polarization rotator consists of a birefringent structure (BFS) with rotated quarter-wave plates $\left(+45^{\circ}\right.$ and $-45^{\circ}$ rotated $)$ on either side. The BFS consists of two tunable birefrigent boards (Board 1 and Board $_{2}$ ). The distances between the boards are $l_{1}=1.66 \mathrm{~mm}$ and $l_{2}=3.26 \mathrm{~mm}$.

$$
\mathbf{Q}_{\theta}=\mathbf{R}(\theta) \mathbf{Q} \mathbf{R}^{T}(\theta),
$$

where $\mathbf{R}(\theta)$ is the rotation matrix:

$$
\mathbf{R}(\theta)=\left[\begin{array}{cc}
\cos \theta & -\sin \theta \\
\sin \theta & \cos \theta
\end{array}\right]
$$

Thus, the Jones matrices for the $+45^{\circ}$ and $-45^{\circ}$ rotated QWPs are

$$
\begin{aligned}
\mathbf{Q}_{+45^{\circ}} & =\mathbf{R}\left(+45^{\circ}\right) \mathbf{Q} \mathbf{R}^{T}\left(+45^{\circ}\right) \\
& =e^{j \alpha}\left[\begin{array}{cc}
\frac{1}{\sqrt{2}} & -\frac{1}{\sqrt{2}} \\
\frac{1}{\sqrt{2}} & \frac{1}{\sqrt{2}}
\end{array}\right]\left[\begin{array}{cc}
1 & 0 \\
0 & e^{j \pi / 2}
\end{array}\right]\left[\begin{array}{cc}
\frac{1}{\sqrt{2}} & \frac{1}{\sqrt{2}} \\
-\frac{1}{\sqrt{2}} & \frac{1}{\sqrt{2}}
\end{array}\right] \\
& =\frac{e^{j \alpha} e^{j(\pi / 4)}}{\sqrt{2}}\left[\begin{array}{cc}
1 & -j \\
-j & 1
\end{array}\right], \\
\mathbf{Q}_{-45^{\circ}} & =\mathbf{R}\left(-45^{\circ}\right) \mathbf{Q} \mathbf{R}^{T}\left(-45^{\circ}\right) \\
& =e^{j \alpha}\left[\begin{array}{cc}
\frac{1}{\sqrt{2}} & \frac{1}{\sqrt{2}} \\
-\frac{1}{\sqrt{2}} & \frac{1}{\sqrt{2}}
\end{array}\right]\left[\begin{array}{cc}
1 & 0 \\
0 & e^{j \pi / 2}
\end{array}\right]\left[\begin{array}{cc}
\frac{1}{\sqrt{2}} & -\frac{1}{\sqrt{2}} \\
\frac{1}{\sqrt{2}} & \frac{1}{\sqrt{2}}
\end{array}\right] \\
& =\frac{e^{j \alpha} e^{j(\pi / 4)}}{\sqrt{2}}\left[\begin{array}{cc}
1 & j \\
j & 1
\end{array}\right] .
\end{aligned}
$$

The BFS is a transmissive metasurface with independently tunable phase shift for both $x$ and $y$ polarizations. The Jones matrix for the BFS can be expressed as 


$$
\mathbf{B}=e^{j \beta}\left[\begin{array}{cc}
1 & 0 \\
0 & e^{j \delta}
\end{array}\right]
$$

Here, $\beta$ is the common transmission phase, and $\delta$ is the transmission phase difference between the two polarizations. The Jones matrix of the entire polarization rotator is simply a product of the Jones matrices of the constitutive elements:

$$
\begin{aligned}
\mathbf{P} & =\mathbf{Q}_{-\mathbf{4 5}^{\circ}} \mathbf{B}_{+\mathbf{4 5}^{\circ}} \\
& =e^{j[2 \alpha+(\pi / 2)+\beta+(\delta / 2)]}\left[\begin{array}{cc}
\cos \left(\frac{\delta}{2}\right) & -\sin \left(\frac{\delta}{2}\right) \\
\sin \left(\frac{\delta}{2}\right) & \cos \left(\frac{\delta}{2}\right)
\end{array}\right] .
\end{aligned}
$$

Comparing the Jones matrix of the polarization rotator with the rotation matrix shown in Eq. (3), it is clear that the proposed device can rotate the polarization of a wave by a rotation angle $\theta_{r}=(\delta / 2)$. The phase difference $\delta$ will be tuned through a bias voltage applied to the BFS. Ideally, we would like $\delta$ to have a tunable range of $360^{\circ}$. This would allow $\theta_{r}$ to be tuned over a range of $180^{\circ}$. Note that if $\beta$ and $\delta$ can be independently tuned for each pixel, the proposed polarization rotator could potentially provide simultaneous wave front shaping and global polarization rotation.

\section{DESIGN AND SIMULATION OF A TUNABLE POLARIZATION ROTATOR}

In this section, we propose and describe metasurface designs for the QWPs and BFS that comprise the polarization rotator. Furthermore, numerical simulation results for the QWPs, BFS, as well as the entire polarization rotator are reported.

\section{A. QWPs: Design and numerical simulation results}

The design of the QWP is based on that reported in Ref. [30]. The topology of the designed QWP is shown in Fig. 2(a). The design consists of three anisotropic admittance sheets realized as patterned metallic claddings. In order to achieve full transmission for both polarizations (i.e., $\hat{x}$ and $\hat{y}$ ) at normal incidence, the two outer sheets should be identical; the QWP should be symmetric. The sheet admittance of the outer and inner sheets can be expressed as $\mathbf{Y}_{\mathbf{S 1}}$ and $\mathbf{Y}_{\mathbf{S 2}}$ :

$$
\begin{aligned}
& \mathbf{Y}_{\mathbf{S 1}}=\left[\begin{array}{cc}
Y_{s 1}^{x x} & 0 \\
0 & Y_{s 1}^{y y}
\end{array}\right], \\
& \mathbf{Y}_{\mathbf{S 2}}=\left[\begin{array}{cc}
Y_{s 2}^{x x} & 0 \\
0 & Y_{s 2}^{y y}
\end{array}\right] .
\end{aligned}
$$

Here, $Y_{s 1}^{x x}$ and $Y_{s 2}^{x x}$ represent the outer and inner sheet admittances for $x$-polarized plane waves, and $Y_{s 1}^{y y}$ and $Y_{s 2}^{y y}$

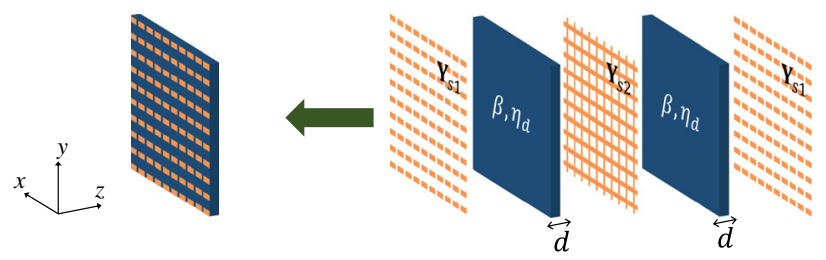

(a)
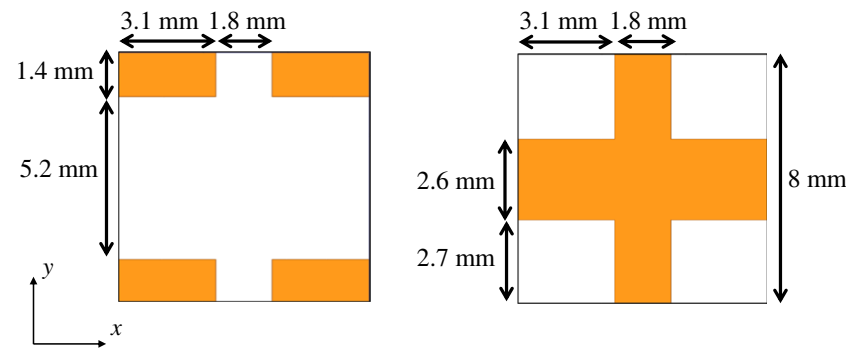

(b)

FIG. 2. (a) Topology of the QWP. The design uses Rogers 4003C substrates $\left(\varepsilon_{r}=3.55\right.$ and $\left.\tan \delta=0.0027\right)$ with a thickness of $d=1.524 \mathrm{~mm}$. (b) Metallic patterns on the outer (left) and inner (right) sheets of the designed QWP.

represent the outer and inner sheet admittances for $y$-polarized plane waves. The sheet admittances can be determined by stipulating a $90^{\circ}$ phase difference between the $x$ and $y$ polarization, near unity transmission amplitude for both polarizations, and a common phase delay $\alpha$. For the designed QWP, the substrates are chosen to be Rogers 4003C $\left(\epsilon_{r}=3.55\right.$ and $\left.\tan \delta=0.0027\right)$, with a thickness of $d=1.524 \mathrm{~mm}$ each. For a given common phase delay of $\alpha$ at the operating frequency of $10 \mathrm{GHz}$, the admittances can be calculated (see Appendix A for details.). The metallic patterns of the inner and outer sheets of the QWP are designed to realize the calculated admittance values of $\mathbf{Y}_{\mathbf{S 1}}$ and $\mathbf{Y}_{\mathbf{S 2}}$. The unit cell dimensions are shown in Fig. 2(b).

The commercial electromagnetic solver ANSYS HFSS was used to design the metallic patterns and simulate the performance of the metasurfaces. For an incident field that is linearly polarized in the $(\hat{x} \pm \hat{y}) / \sqrt{2}$ direction, the simulation results for the designed QWP are shown in Fig. 3. Note that, throughout the paper, the efficiency is

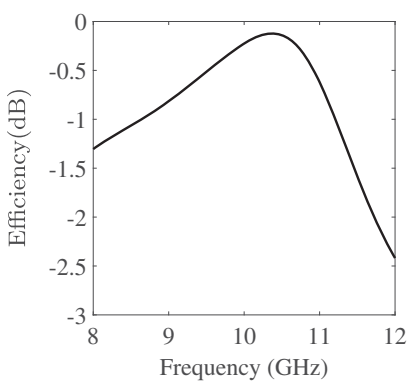

(a)

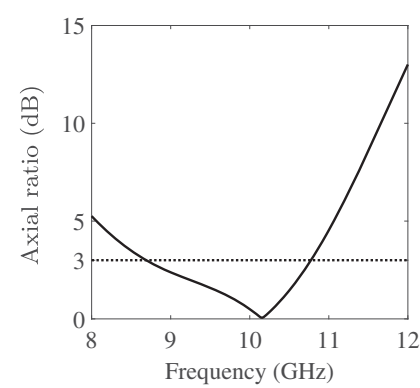

(b)
FIG. 3. Simulation results of (a) efficiency and (b) axial ratio of the designed QWP. 
defined as the ratio of the transmitted power to the incident power. For instance, the efficiency is $\left(\left|S_{21}^{x x}+S_{21}^{x y}\right|^{2}+\mid S_{21}^{y y}+\right.$ $\left.\left.S_{21}^{y x}\right|^{2}\right) / 2$ for an $(\hat{x} \pm \hat{y}) / \sqrt{2}$ polarized incident wave (see Fig. 3). For an $x$-polarized incident wave, the efficiency is defined as $\left(\left|S_{21}^{x x}\right|^{2}+\left|S_{21}^{y x}\right|^{2}\right)$. For a $y$-polarized incident wave, the efficiency is defined as $\left(\left|S_{21}^{x y}\right|^{2}+\left|S_{21}^{y y}\right|^{2}\right)$. Theoretically, the QWP transforms a linearly polarized incident wave to a circularly polarized transmitted wave. A wave is considered circularly polarized if its axial ratio is less than $3 \mathrm{~dB}$. Figure 3(b) shows that the designed QWP has a $3 \mathrm{~dB}$ axial ratio bandwidth of approximately $20 \%$. The efficiency of the QWP is higher than $-3 \mathrm{~dB}$ over the entire $X$ band $(8-12 \mathrm{GHz})$.

\section{B. Birefringent structure: Design and numerical simulation results}

In the design of the BFS, the intent is to realize a broadband transmissive metasurface with a tunable phase delay for both $x$ and $y$ polarizations. The BFS consists of two cascaded birefringent boards [i.e., Board ${ }_{1}$ and Board 2 shown in Fig. 4(a)], rotated $90^{\circ}$ with respect to each other. Each board operates as a tunable phase shifter for the electric field polarization directed along the varactor diodes (MAVR-000120-1411 from MACOM [31]). We refer to the polarization along the diodes as the "parallel polarization," and refer to the orthogonal polarization as "perpendicular

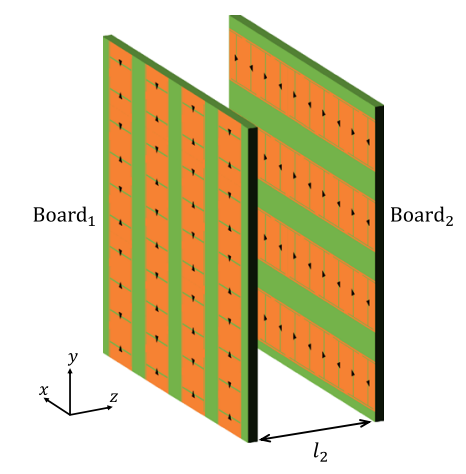

(a)
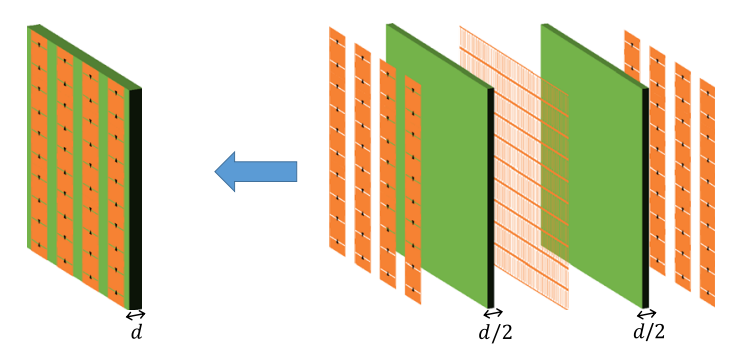

(b)

FIG. 4. (a) Topology of the BFS consisting of two birefringent boards rotated $90^{\circ}$ with respect to each other $\left(l_{2}=3.26 \mathrm{~mm}\right)$. (b) Each birefringent board consists of three patterned metal sheets. The sheets are cascaded with substrates of overall thickness of $d=3.15 \mathrm{~mm}$. polarization." For the parallel polarization, the birefringent board maintains a high transmission across the variable phase delay range. For the perpendicular polarization, the birefringent board still maintains a high transmission while maintaining a fixed phase delay.

A circuit model of the phase shifter for the parallel polarization is shown in Fig. 5(a). It consists of a quarter wavelength of free-space transmission line separating two shunt $L C$ resonators. The resonant frequency of the resonators is $10 \mathrm{GHz}$. The quarter-wavelength line converts the second shunt resonant circuit, to a series resonant circuit, resulting in the equivalent circuit model shown in Fig. 5(b). The effective capacitance $C_{\alpha}$ and inductance $L_{\alpha}$ can be easily calculated as (see Ref. [32])

$$
L_{\alpha}=C Z_{0}^{2}, \quad C_{\alpha}=\frac{L}{Z_{0}^{2}},
$$

where $Z_{0}=377 \Omega$ is the free-space wave impedance. The phase shifter shown in Fig. 5(b) provides a relatively linear phase variation and an impedance match over a wide frequency range [33]. An impedance match is also maintained over a wide range of capacitance $C$ values. This can be easily shown by calculating the circuit's image impedance and wave number [32].

However, the problem with the circuit shown in Fig. 5(a) is that it is bulky, given that it employs a quarter

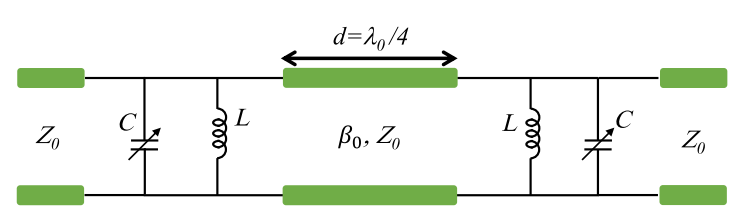

(a)

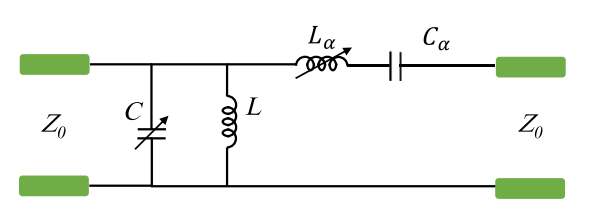

(b)

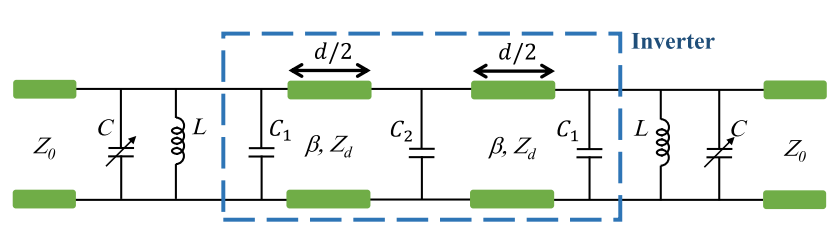

(c)

FIG. 5. (a) A tunable, transmission-line phase shifter that maintains an impedance match (low insertion loss) over its tunable range. (b) Equivalent circuit of the phase shifter shown in Fig. 5(a). (c) A tunable phase shifter employing an impedance inverter instead of a quarter-wavelength transmission line. 
wavelength of free-space transmission line. For miniaturization purposes, the quarter-wavelength, free-space transmission line was replaced with the inverter design shown in Fig. 5(c). The $A B C D$ (transmission) matrix of a quarterwavelength, free-space transmission line is

$$
\left[\begin{array}{ll}
A & B \\
C & D
\end{array}\right]_{\text {free space }}=\left[\begin{array}{cc}
0 & -j Z_{0} \\
-j \frac{1}{Z_{0}} & 0
\end{array}\right] .
$$

On the other hand, the $A B C D$ matrix of the inverter [see Fig. 5(c)] can be written as

$$
\begin{aligned}
{\left[\begin{array}{ll}
A & B \\
C & D
\end{array}\right]_{\text {inverter }}=} & {\left[\begin{array}{cc}
1 & 0 \\
j \omega C_{1} & 1
\end{array}\right]\left[\begin{array}{cc}
\cos \frac{\beta d}{2} & j Z_{d} \sin \frac{\beta d}{2} \\
j \frac{1}{Z_{d}} \sin \frac{\beta d}{2} & \cos \frac{\beta d}{2}
\end{array}\right] } \\
& \times\left[\begin{array}{cc}
1 & 0 \\
j \omega C_{2} & 1
\end{array}\right]\left[\begin{array}{cc}
\cos \frac{\beta d}{2} & j Z_{d} \sin \frac{\beta d}{2} \\
j \frac{1}{Z_{d}} \sin \frac{\beta d}{2} & \cos \frac{\beta d}{2}
\end{array}\right] \\
& \times\left[\begin{array}{cc}
1 & 0 \\
j \omega C_{1} & 1
\end{array}\right]
\end{aligned}
$$

Setting the two $A B C D$ matrices above equal to each other, the following expressions for $C_{1}$ and $C_{2}$ can be obtained:

$$
\begin{aligned}
& C_{1}=\frac{\cos \beta d-\frac{1}{2} Z_{d} \omega C_{2} \sin \beta d}{Z_{d} \omega \sin 2 \beta d-Z_{d}^{2} \omega^{2} C_{2} \sin ^{2} \frac{\beta d}{2}}, \\
& C_{2}=\frac{Z_{0}+Z_{d} \sin \beta d}{Z_{d}^{2} \omega \sin ^{2} \frac{\beta d}{2}} .
\end{aligned}
$$

In the design of the BFS, an RT/Duroid 5880 substrate $\left(\varepsilon_{r}=2.2, \tan \delta=0.0009\right.$, and $\left.d / 2=1.575 \mathrm{~mm}\right)$ from Rogers Corporation was used. Using Eqs. (12) and (13), the values of $C_{1}$ and $C_{2}$ were calculated to be $C_{1}=$ $0.160 \mathrm{pF}$ and $C_{2}=0.656 \mathrm{pF}$. By replacing the quarterwavelength transmission line with the designed inverter, the thickness of the birefringent board was reduced from 7.5 to $3.15 \mathrm{~mm}$. This miniaturization came at the cost of a slight increase in conductor losses and reduced bandwidth.

The circuit model shown in Fig. 5(c) can be realized by three patterned metallic sheets, as shown in Fig. 4(b). The outer sheets of each board have the same metallic pattern, and are loaded with varactor diodes, as shown in Fig. 6(a). In order to simplify the design, the capacitance $C_{1}$ was incorporated into the capacitance of the varactors. For the parallel polarization, the varactor loaded strips and inductive wires provide capacitive and inductive responses, respectively. As a result, the outer sheet of each unit cell behaves as a shunt $L C$ resonator. The inner sheet of each board provides the required capacitance $C_{2}$ for the parallel polarization, as shown in Fig. 6(b). The sheet impedance of the inner sheet was calculated to be $Z_{\text {inner }}^{\|}=\left[1 /\left(j \omega C_{2}\right)\right]=$ $-j 24.23 \Omega$, where the superscript "||" refers to the parallel polarization.

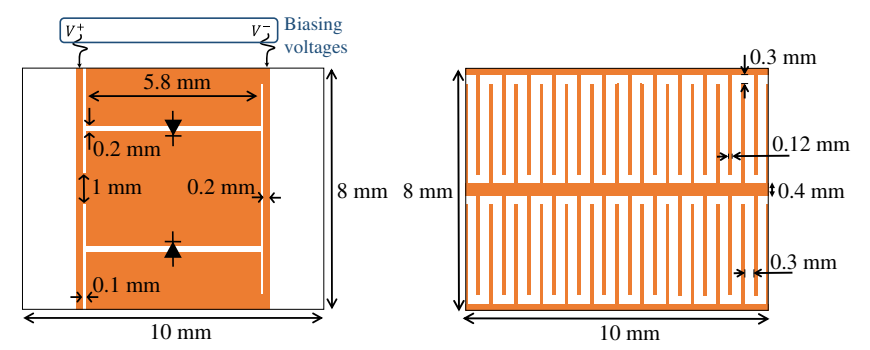

(a)

(b)

FIG. 6. Dimensions of unit cells of the birefringent board. (a) Outer sheet and (b) inner sheet patterns.

Several considerations were taken into account when designing the outer sheet. The inductive lines, in addition to providing the required inductive response for the metasurface's microwave performance, serve as a dc voltage bias network for the varactor diodes. The width of the inductive lines was chosen to provide the highest achievable inductance value for $L$, given the fabrication limitations. The parallel $L C$ circuit shown in Fig. 5(a) has a $Q$ factor that decreases with increasing values of $L$, for a fixed resonant frequency. Thus, it exhibits the widest possible bandwidth given the fabrication constraints. Varactor diodes (MAVR-000120-141) were chosen to provide the required capacitance range. The SPICE model of the varactor as well as its capacitance and resistance performances are provided in its data sheet [31]. An antiseries varactor pair was used for parallel biasing the variable capacitor. In addition, the antiseries configuration provides second and third harmonic suppression, and as a result reduces distortion [34]. Note that the unit cell size used for the birefringent board is $8 \times 10 \mathrm{~mm}^{2}(0.27 \lambda \times 0.33 \lambda)$, which is slightly larger than those typically used in metasurface designs (approximately $0.1 \lambda$ ). This unit cell size was chosen to reduce the required number of varactor diodes.

For the perpendicular polarization, the impedance of the outer sheet was extracted from simulation for the unit cell dimensions shown in Fig. 6(a): $Z_{\text {outer }}^{\perp}=-j 238.01 \Omega$, where the superscript " $\perp$ " refers to the perpendicular polarization. The $A B C D$ matrix of the birefringent board for the perpendicular polarization can be calculated as

$$
\begin{aligned}
{\left[\begin{array}{ll}
A & B \\
C & D
\end{array}\right]=} & {\left[\begin{array}{cc}
1 & 0 \\
\frac{1}{Z_{\text {outer }}^{\perp}} & 1
\end{array}\right]\left[\begin{array}{cc}
\cos \frac{\beta d}{2} & j Z_{d} \sin \frac{\beta d}{2} \\
j \frac{1}{Z_{d}} \sin \frac{\beta d}{2} & \cos \frac{\beta d}{2}
\end{array}\right] } \\
& \times\left[\begin{array}{cc}
1 & 0 \\
\frac{1}{Z_{\text {inner }}^{\perp}} & 1
\end{array}\right]\left[\begin{array}{cc}
\cos \frac{\beta d}{2} & j Z_{d} \sin \frac{\beta d}{2} \\
j \frac{1}{Z_{d}} \sin \frac{\beta d}{2} & \cos \frac{\beta d}{2}
\end{array}\right] \\
& \times\left[\begin{array}{cc}
1 & 0 \\
\frac{1}{Z_{\text {outer }}^{\perp}} & 1
\end{array}\right]
\end{aligned}
$$

The transmission coefficient of the birefringent board for the perpendicular polarization reads 


$$
S_{21}^{\perp}=\frac{2}{A+\frac{B}{\eta_{0}}+C \eta_{0}+D}
$$

Setting $\left|S_{21}^{\perp}\right|=1$, the impedance of the inner sheet for the perpendicular polarization was obtained: $Z_{\text {inner }}^{\perp}=j 89.89 \Omega$.

So far, we have determined the sheet impedances of the inner sheet for both polarizations. The metallic patterns of the inner sheet were designed to realize these sheet impedances [see Fig. 6(b)]. Interdigitated metallic patterns were designed for the parallel polarization and thin metal traces for the perpendicular polarization to provide the required large capacitance and large inductance, respectively. Once the metallic patterns were determined, the entire cascaded birefringent board was simulated, and the dimensions were slightly adjusted to compensate for nearfield coupling.

Simulation results demonstrating the tunability of the birefringent board are given in Fig. 7. In the ANSYS HFSS simulations, each varactor diode was modeled as a lumped capacitance in series with a resistance. The varactor capacitance and resistance characteristic used in the simulation is given in Table I. The characteristic was extracted from its SPICE model [31] simulated at $10 \mathrm{GHz}$. The plots show that the transmission amplitude for the perpendicular polarization for all diode capacitances is around $\left|S_{21}^{\perp}\right|=-0.36 \mathrm{~dB}$. The transmission amplitude for the parallel polarization remains above $-3 \mathrm{~dB}$ at the operating frequency $(10 \mathrm{GHz})$,

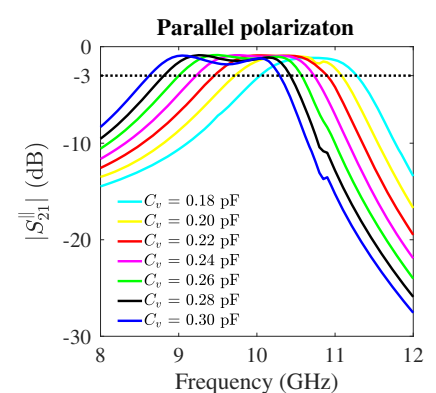

(a)

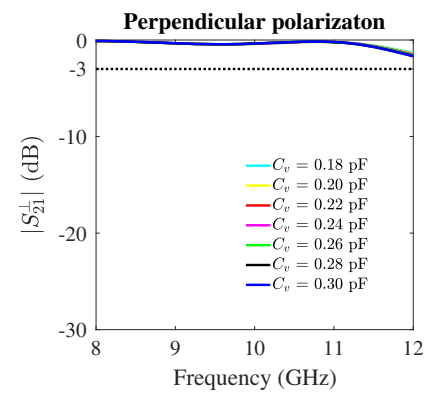

(c)

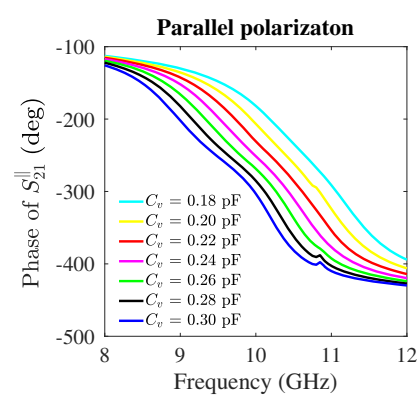

(b)

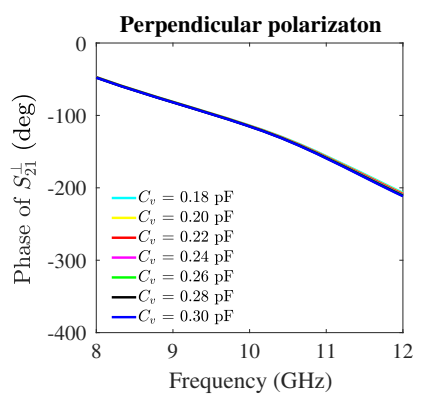

(d)
FIG. 7. Simulated transmission response of the birefringent board. (a) Transmission amplitude for the parallel polarization. (b) Transmission phase for the parallel polarization. (c) Transmission amplitude for the perpendicular polarization. (d) Transmission phase in the perpendicular polarization.
TABLE I. Varactor (MAVR-000120-1411) capacitance and resistance verses reverse bias voltage, extracted from the SPICE model.

\begin{tabular}{lcc}
\hline \hline $\begin{array}{l}\text { Reverse bias } \\
\text { voltage }(\mathrm{V})\end{array}$ & $\begin{array}{c}\text { Varactor } \\
\text { capacitance }(\mathrm{pF})\end{array}$ & $\begin{array}{c}\text { Varactor } \\
\text { resistance }(\Omega)\end{array}$ \\
\hline 5.9 & 0.30 & 0.25 \\
6.5 & 0.28 & 0.22 \\
7.4 & 0.26 & 0.19 \\
8.3 & 0.24 & 0.16 \\
9.6 & 0.22 & 0.12 \\
11.6 & 0.20 & 0.08 \\
14.7 & 0.18 & 0.04 \\
\hline \hline
\end{tabular}

as the capacitance of the diodes is varied between 0.18 and $0.30 \mathrm{pF}$. At the operating frequency, the maximum transmission amplitude $\left(\left|S_{21}^{\|}\right|=-0.91 \mathrm{~dB}\right)$ for the parallel polarization occurs at $C_{v}=0.22 \mathrm{pF}$. The transmission phase for this capacitance range can be varied from $-303.2^{\circ}$ to $-181.8^{\circ}$, providing a maximum phase range of $121.4^{\circ}$ for the parallel polarization. Thus, the phase difference between the two polarizations of the BFS (with Board $_{1}$ and Board 2 cascaded together) can be varied from $\delta=-121.4^{\circ}$ to $\delta=121.4^{\circ}$.

In addition, for the parallel polarization, the extracted circuit model with detailed component values of the designed birefringent board is shown in Appendix B. The frequency response of the circuit model and the full-wave simulation results of the birefringent board agree closely.

\section{Polarization rotator (cascaded structure): Numerical simulation results}

As shown in Fig. 1, the polarization rotator is a cascaded structure consisting of an electrically tunable BFS and two $\pm 45^{\circ}$ rotated QWPs. Based on the simulation results of the QWPs and birefringent boards presented in the previous sections, the simulated performance of the entire polarization rotator can be obtained by cascading the simulation results of all its constitutive components. It should be noted that near-field coupling effects between different boards are neglected in the simulation.

The rotation angle $\theta_{r}$ of the polarization rotator can be tuned with the bias voltage, which controls the birefringence of the BFS. We define the varactor capacitances in the $x$ and $y$ directions as $C_{x}$ and $C_{y}$, respectively. Based on the configuration shown in Fig. $1, C_{y}$ is the varactor capacitance of Board ${ }_{1}$ and $C_{x}$ the varactor capacitance of Board $_{2}$. The capacitances, $C_{x}$ and $C_{y}$, can vary from 0.18 to $0.30 \mathrm{pF}$. The polarization rotator was excited with linearly polarized waves in the $x$ and $y$ directions. The simulated rotation angle $\theta_{r}$ versus varactor capacitance is shown in Tables II and III for $x$-polarized and $y$-polarized incident waves, respectively. A comparison of Tables II and III shows that, for the same $C_{x}$ and $C_{y}$ combination, the values 
TABLE II. Simulated $\theta_{r}$ (degrees) for an $x$-polarized incident wave (at $10 \mathrm{GHz}$ ).

\begin{tabular}{l|c|cccccc}
\hline \hline \multicolumn{2}{c|}{} & \multicolumn{6}{|c}{$C_{x}(\mathrm{pF})$} \\
\cline { 3 - 8 } \multicolumn{2}{l|}{} & 0.18 & 0.2 & 0.22 & 0.24 & 0.26 & 0.30 \\
\hline \multirow{4}{*}{$C_{y}(\mathrm{pF})$} & 0.18 & $\mathbf{- 0 . 0 1 6}$ & $\mathbf{1 0 . 5 3}$ & $\mathbf{2 5 . 6}$ & $\mathbf{4 1 . 0 4}$ & $\mathbf{5 2 . 4 8}$ & $\mathbf{6 8 . 8 5}$ \\
& 0.2 & $\mathbf{- 9 . 5 6}$ & $\mathbf{0 . 0 1 0}$ & $13.82^{\mathrm{a}}$ & 27.91 & 38.73 & 54.98 \\
& 0.22 & -21.8 & -12.68 & 0.015 & 13.01 & 23.27 & 39.44 \\
& 0.24 & -32.84 & -24.3 & -12.31 & 0.003 & 9.89 & 25.99 \\
& 0.26 & -41.87 & -33.48 & -21.72 & -9.686 & 0.001 & 15.98 \\
& 0.30 & $\mathbf{- 5 8 . 2 6}$ & -49.57 & -37.42 & -25.26 & -15.68 & 0.006 \\
\hline \hline
\end{tabular}

${ }^{a}$ Rotation angle value in standard font refers to an efficiency larger than $-3 \mathrm{~dB}$, and an axial ratio larger than $10 \mathrm{~dB}$.

${ }^{b}$ Rotation angle value in bold font refers to an efficiency less than $-3 \mathrm{~dB}$, and an axial ratio larger than $10 \mathrm{~dB}$.

TABLE III. Simulated $\theta_{r}$ (degrees) for a $y$-polarized incident wave (at $10 \mathrm{GHz}$ ).

\begin{tabular}{c|c|cccccc}
\hline \hline \multicolumn{2}{c|}{} & \multicolumn{6}{|c}{$C_{x}(\mathrm{pF})$} \\
\cline { 3 - 8 } \multicolumn{2}{l|}{} & 0.18 & 0.2 & 0.22 & 0.24 & 0.26 & 0.30 \\
\hline \multirow{5}{*}{$C_{y}(\mathrm{pF})$} & 0.18 & $\mathbf{- 0 . 0 0 7}$ & $\mathbf{1 1 . 8 2}$ & $24.69^{\mathrm{a}}$ & 35.77 & 45.23 & $\mathbf{5 9 . 2 3}$ \\
& 0.2 & $\mathbf{- 1 2 . 5 8}$ & $\mathbf{0 . 0 0 8}$ & 14.09 & 26.24 & 35.33 & 50.67 \\
& 0.22 & $\mathbf{- 2 8 . 8 9}$ & -15.33 & 0.000 & 13.12 & 22.69 & 38.08 \\
& 0.24 & $\mathbf{- 4 3 . 9}$ & -29.94 & -13.82 & 0.004 & 9.937 & 25.38 \\
& 0.26 & $\mathbf{- 5 4 . 3 9}$ & -40.57 & -24.23 & -10.13 & -0.001 & 15.64 \\
& 0.30 & $\mathbf{- 6 9 . 2 7}$ & -56.03 & -40.1 & -26.13 & -15.97 & -0.006 \\
\hline \hline
\end{tabular}

${ }^{a}$ Rotation angle value in standard font refers to an efficiency larger than $-3 \mathrm{~dB}$, and an axial ratio larger than $10 \mathrm{~dB}$.

${ }^{b}$ Rotation angle value in bold font refers to an efficiency less than $-3 \mathrm{~dB}$, and an axial ratio larger than $10 \mathrm{~dB}$.

of $\theta_{r}$ at $10 \mathrm{GHz}$ are very close for $x$ - and $y$-polarized incident waves. The simulated frequency performance of the polarization rotator for $x$ - and $y$-polarized incident waves is shown in Fig. 8. Cases are shown where the

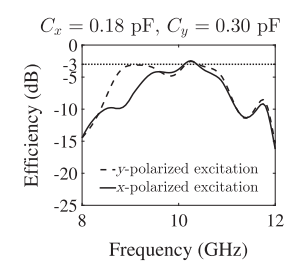

(a)

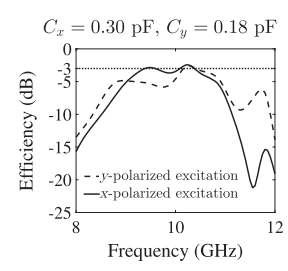

(d)

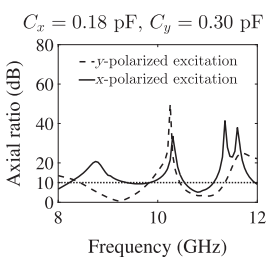

(b)

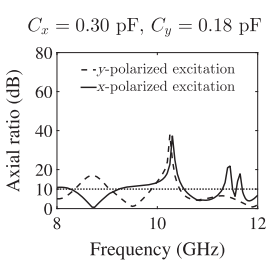

(e)

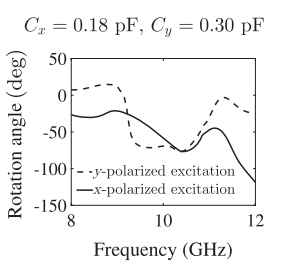

(c)

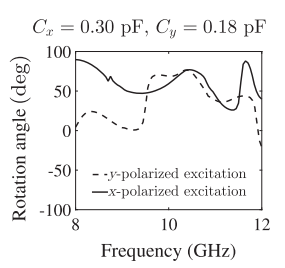

(f)
FIG. 8. Simulation results for the polarization rotator under $x$ - and $y$-polarized incident wave.
TABLE IV. Simulated performance.

\begin{tabular}{lc}
\hline \hline BFS $\delta$ range & $-121.4^{\circ}$ to $121.4^{\circ}$ \\
Polarization rotator $\theta_{r}$ range & $-69.27^{\circ}$ to $59.23^{\circ}$ \\
Total tunable range of $\theta_{r}$ & $128.5^{\circ}$ \\
\hline \hline
\end{tabular}

varactor capacitances on the two birefringent boards differ the most, i.e., $\left(C_{x}=0.18 \mathrm{pF}, C_{y}=0.30 \mathrm{pF}\right)$ and $\left(C_{x}=\right.$ $\left.0.30 \mathrm{pF}, C_{y}=0.18 \mathrm{pF}\right)$. In Fig. 8 , we see close agreement between the simulation results for $x$ - and $y$-polarized incident waves around the operating frequency. Given that the polarization rotator can rotate both $x$ - and $y$-polarized incident waves with very similar $\theta_{r}$, it is capable of rotating the polarization of arbitrarily polarized incident waves. Hereafter, for the sake of brevity, the simulation and measurement results of the polarization rotator are reported only for $y$-polarized incident waves.

A wave will be considered linearly polarized if its axial ratio is above $10 \mathrm{~dB}$. At the operating frequency of $10 \mathrm{GHz}$, Table III shows that the structure is capable of providing rotation angles ranging from $\theta_{r}=-69.27^{\circ}$ to $\theta_{r}=59.23^{\circ}$, resulting in $128.5^{\circ}$ of tunable rotation angle.

A summary of the polarization rotator's simulated performance is given in Table IV. The results presented in Table IV are at the operating frequency of $10 \mathrm{GHz}$. Note that the simulated rotation angle $\theta_{r}$ range is larger than what is theoretically predicted by the BFS $\delta$ range, due to slight differences between the simulated and theoretical performances of the QWP and BFS at $10 \mathrm{GHz}$.

\section{TUNABLE METASURFACE-BASED POLARIZATION ROTATOR: FABRICATION AND MEASUREMENT}

This section describes the measurement setup used in experiment. Measurements of the QWP and the birefringent board are reported, as well as those for the entire polarization rotator.

\section{A. Measurement setup}

The QWPs and birefringent boards were experimentally characterized using the quasioptical Gaussian beam telescope shown in Fig. 9 [35]. In the experimental setup, the fabricated samples are illuminated with a collimated

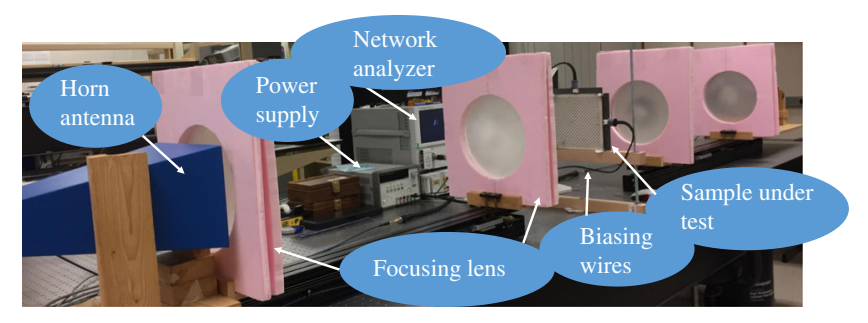

FIG. 9. Photograph of the quasioptical, free-space measurement system. 
Gaussian beam. A rectangular horn antenna (i.e., Dorado GH-90-25) excites the Gaussian beam. A pair of lenses (telescope) focus the Gaussian beam to a beam waist with size and location independent of the operating frequency [36]. The lenses in the telescope are identical, with input and output focal lengths equal to $45 \mathrm{~cm}$. The diameter of the Gaussian beam at its waist is $114 \mathrm{~mm}$. The measured metasurfaces had a diameter that is greater than $168 \mathrm{~mm}$ (about 1.5 times the beam waist), to limit diffraction. The antennas are connected to a vector network analyzer (Agilent E8361A). For further details on this measurement setup, see Ref. [35].

A calibration process is needed to measure the transmission coefficients of metasurfaces. Prior to the measurements, a free-space through, reflect, line calibration was performed for the $y$ polarization. Next, both antennas were rotated by $90^{\circ}$, and the transmission coefficient was measured for $x$ polarization. This measurement characterized path loss (both amplitude and phase) between the two horn antennas for an $x$ polarization. To characterize the rotator, the transmission coefficient between the two antennas was measured for every polarization combination: $S_{21}^{x x}, S_{21}^{x y}, S_{21}^{y x}, S_{21}^{y y}$. The path loss for $x$ polarization was then calibrated out of these measurements. This procedure provided calibrated measurements of the transmission coefficients.

\section{B. QWP: Fabrication and measurement results}

A Rogers 4003C substrate $\left(\epsilon_{r}=3.55\right.$ and $\left.\tan \delta=0.0027\right)$ with a $1 \mathrm{oz}(35 \mu \mathrm{m})$ copper cladding was chosen for the QWP. Rogers 4450F $\left(\epsilon_{r}=3.52\right.$ and $\left.\tan \delta=0.004\right)$ bondply, with a thickness of $0.101 \mathrm{~mm}$, was used as an adhesive layer. A cross section of the material layers used to fabricate the QWP is shown in Fig 10(a). The QWP was fabricated with its optical axes rotated $45^{\circ}$ with respect to the circuit board edges, as shown in Fig. 10(b). The size of the fabricated QWP is $172 \times 172 \mathrm{~mm}^{2}$.

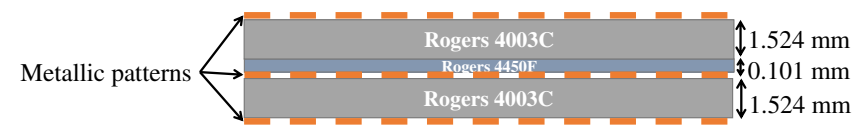

(a)

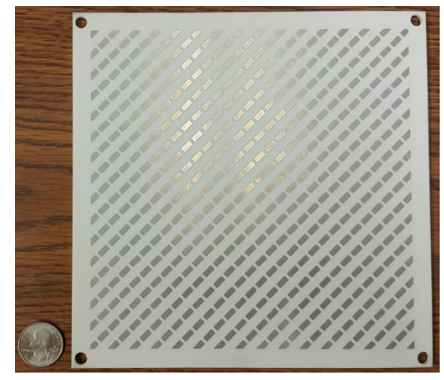

(b)

FIG. 10. (a) Cross section of the QWP. (b) Photograph of the fabricated QWP.

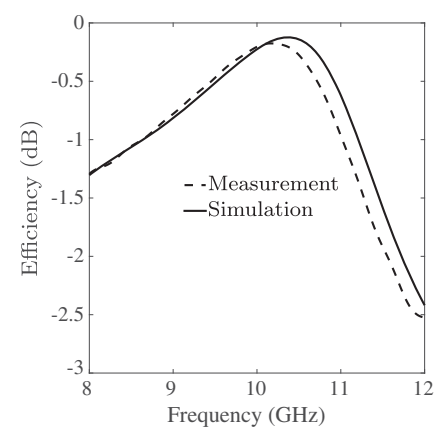

(a)

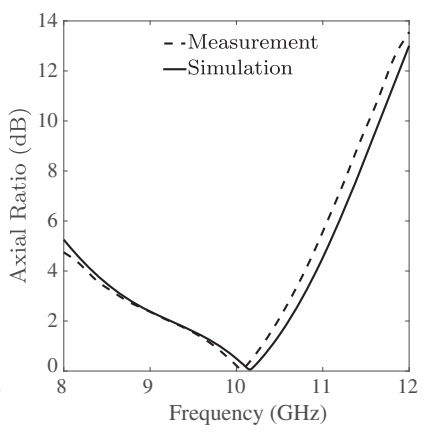

(b)
FIG. 11. Measurement results of (a) efficiency and (b) axial ratio of the fabricated QWP.

Measurement results for the QWP are shown in Fig. 11 for an incident wave linearly polarized in the $y$ direction. Since the Rogers $4450 \mathrm{~F}$ bondply has a small thickness $(0.101 \mathrm{~mm})$ and its permittivity is close to Rogers 4003C substrate, it was neglected in simulation. Simulation and measurement results are in close agreement, with a frequency shift of about $1 \%$ between them.

\section{Tunable BFS: Fabrication and measurement results}

Two birefringent boards (i.e., Board 1 and Board 2 ) with dimensions $170 \times 168 \mathrm{~mm}^{2}$ were fabricated, as shown in Fig. 12(a). The cross section of the birefringent boards is shown in Fig. 12(b). RT/Duroid 5880 substrate was used $\left(\epsilon_{r}=2.2\right.$ and $\left.\tan \delta=0.0009\right)$ together with Rogers 3001 bonding film $\left(\epsilon_{r}=2.28\right.$ and $\left.\tan \delta=0.003\right)$ as the adhesive layer. The thickness of the copper cladding is $35 \mu \mathrm{m}(1 \mathrm{oz})$. Again, the bonding film was neglected in simulation, since

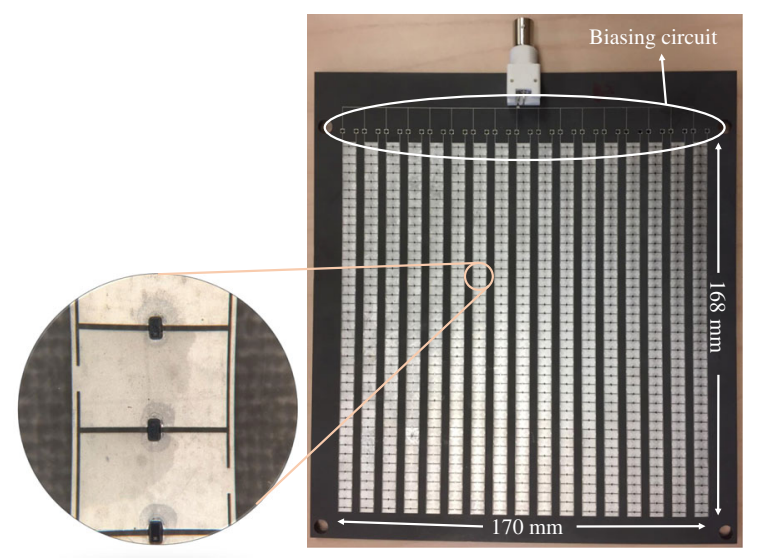

(a)

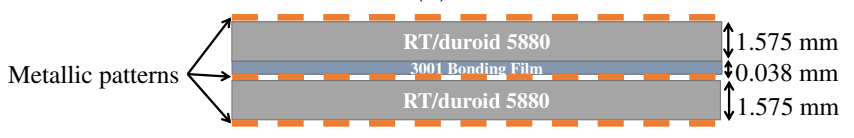

(b)

FIG. 12. (a) Photograph of the fabricated BFS. (b) Cross section of the BFS. 


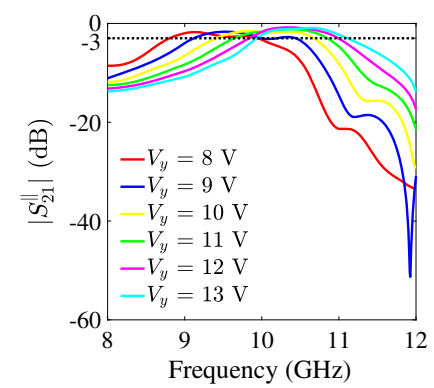

(a)

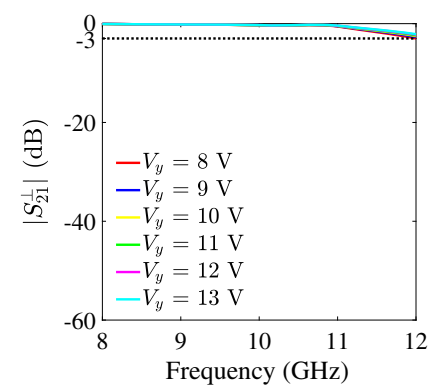

(c)

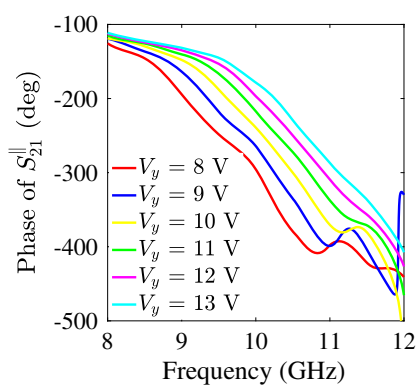

(b)

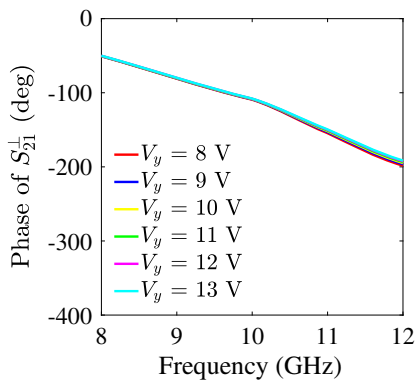

(d)
FIG. 13. Measured transmission response of the birefringent boards. (a) Transmission amplitude for the parallel polarization (y polarization for board 1 and $\mathrm{x}$ polarization for board $_{2}$. (b) Transmission phase for the parallel polarization. (c) Transmission amplitude for the perpendicular polarization (x polarization for board and y polarization for board $_{2}$ ). (d) Transmission phase for the perpendicular polarization.

it has a small thickness $(0.038 \mathrm{~mm})$ and a permittivity close to that of the RT/Duroid 5880 substrate. A total of 2856 MAVR-000120-1411 varactor diodes (diode dimensions $0.4 \times 0.75 \mathrm{~mm}^{2}$ ) were mounted onto each board. As shown in Fig. 12(a), all the unit cells in one column are biased with the same voltage. A gradient in the bias voltages across different columns could allow waveform control. However, in this implementation, we biased all the columns with the same voltage though a BNC connector.

The measurement results for each birefringent board under different biasing voltages are shown in Fig. 13. The varactor capacitance versus reverse bias voltage characteristic quoted on the diode data sheet (as given in Table I) did not accurately match the experimental results. Therefore, the experimental capacitance versus reverse bias voltage relationship of the varactors was fine-tuned by comparing measurement to simulation, as shown in Appendix C. Specifically, the varactor capacitance corresponding to each bias voltage was extracted by aligning the measured phase performance to simulation. The varactor resistances corresponding to the capacitances were taken from Table I. The extracted characteristic of the diodes is shown in Table V.

A minimum bias voltage of $8 \mathrm{~V}$ and maximum bias voltage of $13 \mathrm{~V}$ were chosen to maintain a transmission amplitude above $-3 \mathrm{~dB}$ for the parallel polarization at the
TABLE V. Varactor (MAVR-000120-1411) capacitance at each bias voltage, extracted from measurements.

\begin{tabular}{lc}
\hline \hline Reverse bias voltage $(\mathrm{V})$ & Varactor capacitance $(\mathrm{pF})$ \\
\hline 8 & 0.30 \\
9 & 0.26 \\
10 & 0.24 \\
11 & 0.22 \\
12 & 0.20 \\
13 & 0.18 \\
\hline \hline
\end{tabular}

operating frequency, across the entire bias voltage range. Within this voltage range, the transmission phase difference between the two orthogonal polarizations for a single birefringent board spans $117.8^{\circ}$. Thus, the experimental phase difference of the BFS (with Board 1 and Board cascaded together) given by Eq. (6) can be varied from $\delta=-117.8^{\circ}$ to $\delta=117.8^{\circ}$. Therefore, a maximum rotation angle range of $\theta_{r}=(\delta / 2)=117.8^{\circ}$ is expected for the overall polarization rotator. At the operating frequency, the maximum transmission coefficient of each birefringent board, under a parallel polarization, across all bias voltages, is $\left|S_{21}^{\|}\right|=-1.45 \mathrm{~dB}$. This is approximately $0.54 \mathrm{~dB}$ lower than predicted from simulation. This added loss can be attributed to higher diode loss and extra loss introduced by the tinning and soldering process used to attach the diodes. For the perpendicular polarization, the transmission coefficient across all bias voltages is $-0.32 \mathrm{~dB}$ in magnitude and $-107.9^{\circ}$ in phase, at the operating frequency. These results closely match simulation: a transmission amplitude of $-0.36 \mathrm{~dB}$ and transmission phase of $-115.4^{\circ}$. In general, it can be seen that the simulation and measurement results of the birefringent board are in very good agreement. A detailed comparison between the simulation and measurement results is provided in Appendix C.

\section{Tunable polarization rotator: Measurement results}

The experimental polarization rotator was assembled by cascading the birefringent boards and QWPs (see Fig. 14). Layers of the overall polarization rotator were held together using nylon screws. The distance between the QWPs and BFS is $1.66 \mathrm{~mm}$, and the distance between the two birefringent boards is $3.26 \mathrm{~mm}$. The total thickness of the fabricated polarization rotator is $19 \mathrm{~mm}$, which is approximately $0.6 \lambda$. The spacings between boards that minimize evanescent coupling were determined through experiment. Here, the bias voltages in the $x$ and $y$ directions are denoted as $V_{x}$ and $V_{y}$, respectively. In Fig. $1, V_{x}$ is the bias voltage on Board 2 , and $V_{y}$ is the bias voltage on Board $_{1}$. The bias voltages $V_{x}$ and $V_{y}$ were provided through BNC connectors edge mounted to each birefringent board.

As noted earlier, the rotation angle $\theta_{r}$ of the polarization rotator is controlled by the bias voltages applied to the BFS. 


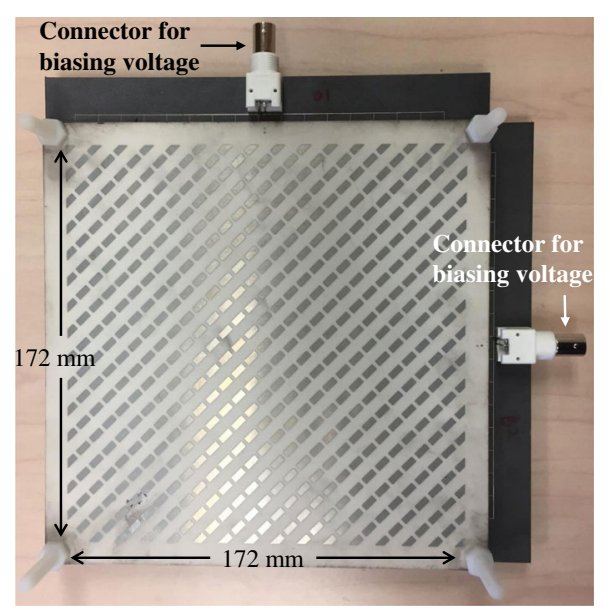

(a)

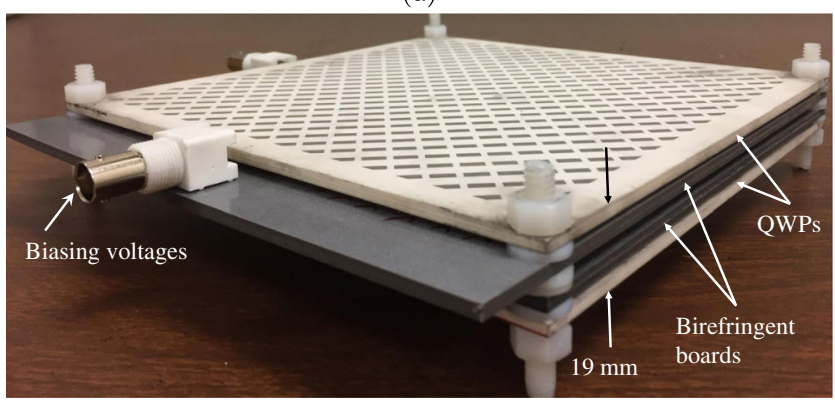

(b)

FIG. 14. Photographs of the fabricated tunable polarization rotator. (a) Top view. (b) Side view.

The bias voltage range used for the BFS was 8-13 V. These bias voltages correspond to varactor capacitances ranging from 0.3 to $0.18 \mathrm{pF}$. Measurement results of the fabricated polarization rotator operating at $10 \mathrm{GHz}$ are shown in Table VI. In these measurements, the incident wave generated by the quasioptical Gaussian beam telescope is linearly polarized in the $y$ direction. The measured axial ratio shows that the transmitted waves are linearly polarized for all bias voltage combinations. The measured results

TABLE VI. Measured $\theta_{r}$ (degrees) of $y$-polarized incident wave (at $10 \mathrm{GHz}$ ).

\begin{tabular}{c|c|cccccc}
\hline \multicolumn{2}{c|}{} & \multicolumn{6}{|c}{$V_{x}(\mathrm{~V})$} \\
\cline { 3 - 8 } \multicolumn{2}{c|}{} & 13 & 12 & 11 & 10 & 9 & 8 \\
\hline \multirow{6}{*}{$V_{y}(\mathrm{~V})$} & 13 & $\mathbf{- 1 . 6 4 1}$ & $\mathbf{9 . 4 2 8}$ & $17.48^{\mathrm{a}}$ & 32.73 & $\mathbf{4 7 . 2 9}$ & $\mathbf{6 9 . 1 8}$ \\
& 12 & $\mathbf{- 1 5 . 0 6}$ & $\mathbf{3 . 2 6 4}$ & 11.44 & 23.88 & 40.26 & $\mathbf{6 1 . 8 4}$ \\
& 11 & $\mathbf{- 3 4 . 6 7}$ & $\mathbf{- 1 9}$ & 3.192 & 12.8 & 28.58 & 50.33 \\
& 10 & $\mathbf{- 5 2 . 1 8}$ & $\mathbf{- 3 8 . 5 8}$ & $\mathbf{- 1 9 . 5 7}$ & -2.72 & 12.38 & $\mathbf{3 2 . 9 9}$ \\
& 9 & $\mathbf{- 6 4 . 7}$ & $\mathbf{- 5 2 . 6 4}$ & $\mathbf{- 3 5 . 6 2}$ & -14.41 & $\mathbf{- 0 . 2 6}$ & $\mathbf{1 9 . 6 4}$ \\
& 8 & $\mathbf{- 7 6 . 9 8}$ & $\mathbf{- 6 7 . 3 5}$ & $\mathbf{- 5 0 . 3 6}$ & -31.48 & $\mathbf{- 1 6 . 3 6}$ & $\mathbf{- 5 . 3 5 5}$ \\
\hline
\end{tabular}

${ }^{\mathrm{a}}$ Rotation angle value in standard font refers to an efficiency larger than $-3 \mathrm{~dB}$, and an axial ratio larger than $10 \mathrm{~dB}$.

${ }^{6}$ Rotation angle value in bold font refers to an efficiency less than $-3 \mathrm{~dB}$, and an axial ratio larger than $10 \mathrm{~dB}$.
TABLE VII. Measured performance.

\begin{tabular}{lc}
\hline \hline BFS $\delta$ range & $-117.8^{\circ}$ to $117.8^{\circ}$ \\
Polarization rotator $\theta_{r}$ range & $-76.98^{\circ}$ to $69.18^{\circ}$ \\
Total tunable range of $\theta_{r}$ & $146.16^{\circ}$ \\
\hline \hline
\end{tabular}

show that the rotation angle of the fabricated polarization rotator can be tuned from $\theta_{r}=-76.98^{\circ}$ to $\theta_{r}=69.18^{\circ}$, with a total range of $146.16^{\circ}$. A summary of the measurement results at $10 \mathrm{GHz}$ is provided in Table VII. Comparing Table VII with Table IV, we can see close agreement between measurement and simulation.

Detailed measurements of the fabricated polarization rotator are given for three representative bias voltage combinations $\left(V_{x}=8 \mathrm{~V}, \quad V_{y}=13 \mathrm{~V} ; \quad V_{x}=11 \mathrm{~V}\right.$, $\left.V_{y}=11 \mathrm{~V} ; V_{x}=13 \mathrm{~V}, V_{y}=8 \mathrm{~V}\right)$ in Fig. 15 . The polarization rotator was measured for $x$-polarized and $y$-polarized incident fields. Close agreement was observed in the efficiency, axial ratio, and rotation angle between measurement and simulation results over $X$-band frequencies. In addition, close agreement is observed between $x$ - and $y$-polarized responses of the metasurface near the operating frequency. The axial ratio behavior shows that the fabricated polarization rotator's performance is slightly frequency shifted from simulation, due to the frequency shift observed in the experimental QWPs. The measured efficiency is around $1 \mathrm{~dB}$ lower than in simulation. For the bias voltage combinations, the efficiency varies between -6 and

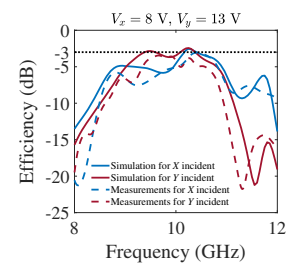

(a)

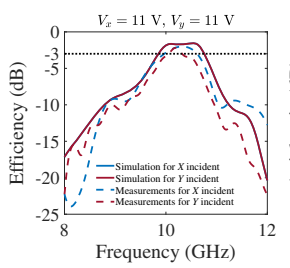

(d)

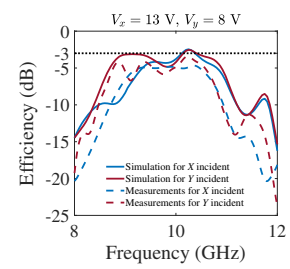

(g)

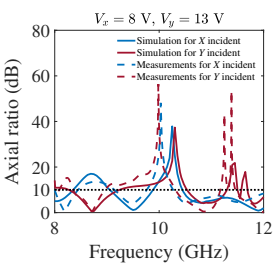

(b)

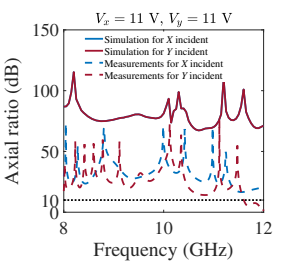

(e)

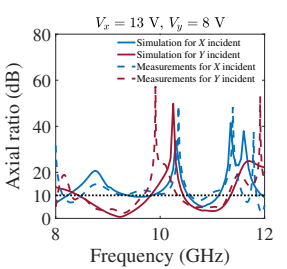

(h)

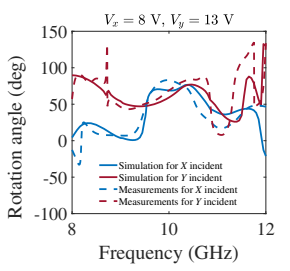

(c)

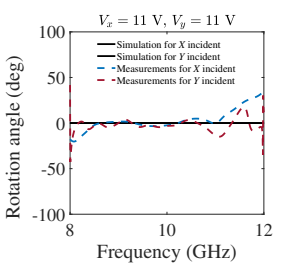

(f)

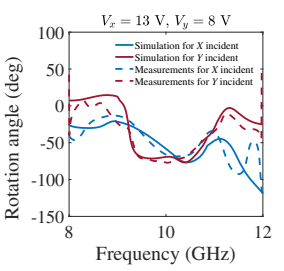

(i)
FIG. 15. Measurement results of efficiency, axial ratio, and rotation angle for the polarization rotator under three extreme biasing voltages combinations. 
$-1.86 \mathrm{~dB}$ at the operating frequency. The lowest efficiencies occur at the minimum and maximum bias voltages. In these cases, the birefringent board exhibits increased reflection. The reflection of each metasurface is reported in Appendix D.

The $10 \mathrm{~dB}$ axial ratio bandwidth of the polarization rotator remains above $6.4 \%$ for all bias voltage combinations. The bandwidth is limited for large rotation angles. Adding extra metallic layers to each constitutive board can improve the bandwidth. This comes at a cost of added loss. In addition, adding extra birefringent boards into the BFS can expand the bandwidth of the polarization rotator. However, doing this will also increase loss, overall thickness, and cost.

\section{CONCLUSION}

We propose a tunable metasurface which can dynamically tune the polarization of an incident wave in transmission. A proof-of-principle metasurface was designed and experimentally demonstrated for the $X$ band. The designed metasurface uses a simple biasing mechanism that does not interfere with the metasurface's microwave performance. At the operating frequency of the structure (i.e., $10 \mathrm{GHz}$ ), the overall rotation angle range achieved by the polarization rotator is $146^{\circ}$, with a reasonably good overall efficiency.

The reported polarization rotator represents a large-scale integration of cascaded metasurfaces. Overall 12 impedance sheets were designed and cascaded together to form four metasurfaces, two of which are reconfigurable. In the end, a compact, quasioptical device was built to implement advanced field control. The designed polarization rotator can provide real-time manipulation of polarization for communication, imaging, and radar systems.

Future research directions include making the metasurface programmable [37-39]. Further, independently biasing columns of the unit cells shown in Fig. 12(a) would enable the polarization rotator to simultaneously rotate polarization and allow beam steering. In addition, as shown in Ref. [40], two phase-discontinuous metasurfaces (inhomogeneous phase-shifting planes) could be used to independently control the amplitude and phase distributions of an aperture field in a lossless and reflectionless manner. Therefore, by cascading two transmissive, phase tunable metasurface, the amplitude and phase distributions of an aperture field could be dynamically controlled.

\section{ACKNOWLEDGMENTS}

This work was supported by DSO National Laboratories under Contract No. DSOCO15027, and the Air Force Office of Scientific Research (AFOSR) under Grant No. FA9550-15-1-0101, and the AFOSR MURI program No. FA9550-18-1-0379.

\section{APPENDIX A: QWP DESIGN DETAILSs}

The design of the QWP is based on the procedure reported in Ref. [30]. The QWP consists of three anisotropic admittance sheets, as shown in Fig. 2(a). The sheet admittances of the outer and inner sheets can be expressed as $\mathbf{Y}_{\mathbf{S} 1}$ and $\mathbf{Y}_{\mathbf{S} 2}$ :

$$
\begin{aligned}
& \mathbf{Y}_{\mathbf{S 1}}=\left[\begin{array}{cc}
Y_{s 1}^{x x} & 0 \\
0 & Y_{s 1}^{y y}
\end{array}\right], \\
& \mathbf{Y}_{\mathbf{S 2}}=\left[\begin{array}{cc}
Y_{s 2}^{x x} & 0 \\
0 & Y_{s 2}^{y y}
\end{array}\right] .
\end{aligned}
$$

Since there are no off-diagonal entries in the sheet admittance $\left(Y^{x y}=Y^{y x}=0\right)$, separate $2 \times 2 A B C D$ matrices can be written for the $x$ and $y$ polarizations. We can calculate the $A B C D$ matrix of the QWP for $x$ polarization by multiplying the corresponding $A B C D$ matrices of its constitutive layers:

$$
\begin{aligned}
{\left[\begin{array}{ll}
A & B \\
C & D
\end{array}\right]=} & {\left[\begin{array}{cc}
1 & 0 \\
Y_{s 1}^{x x} & 1
\end{array}\right]\left[\begin{array}{cc}
\cos \beta d & j \eta_{d} \sin \beta d \\
j \frac{1}{\eta_{d}} \sin \beta d & \cos \beta d
\end{array}\right] . } \\
& \times\left[\begin{array}{cc}
1 & 0 \\
Y_{s 2}^{x x} & 1
\end{array}\right]\left[\begin{array}{cc}
\cos \beta d & j \eta_{d} \sin \beta d \\
j \frac{1}{\eta_{d}} \sin \beta d & \cos \beta d
\end{array}\right] \\
& \times\left[\begin{array}{cc}
1 & 0 \\
Y_{s 1}^{x x} & 1
\end{array}\right] .
\end{aligned}
$$

Given that the dielectric permittivity and the distance between adjacent sheets are $\epsilon_{r}=3.55$ and $d=1.524 \mathrm{~mm}$, respectively, the electrical length between each sheet is $\beta d=(2 \pi) 0.027$. Further, the wave impedance of the dielectric substrate is $\eta_{d}=0.5307 \eta_{0}$, where $\eta_{0}=377 \Omega$ is the wave impedance of the free space.

The transmission coefficient $S_{21}$ of the QWP for $x$ polarization can be calculated from the $A B C D$ matrix as follows:

$$
S_{21}=\frac{2}{A+\frac{B}{\eta_{0}}+C \eta_{0}+D} .
$$

Similarly, the $A B C D$ matrix and corresponding transmission coefficient for $y$ polarization can be calculated. The transmittance $\left(\left|S_{12}\right|^{2}\right)$ and the phase of the transmission coefficient of the QWP are plotted in Fig. 16 for different values of sheet admittances, for a single polarization. The dashed lines in both graphs identify the conditions of unity transmission $\left(\left|S_{12}\right|^{2}=1\right)$.

From Fig. 16(b), we can see that different combinations of sheet admittances $\left(Y_{s 1}\right.$ and $\left.Y_{s 2}\right)$ can provide unity transmission and different transmission phases spanning $360^{\circ}$. By choosing different pairs of $Y_{s 1}$ and $Y_{s 2}$ for each polarization, one can achieve $100 \%$ transmission for both polarizations as well as a $90^{\circ}$ phase difference between the two polarizations. Multiple admittance combinations can be used to achieve the QWP. In our design, the sheet admittances for $x$ and $y$ polarization are given by point $\mathrm{A}$ 


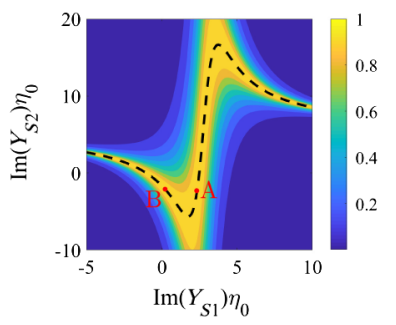

(a)

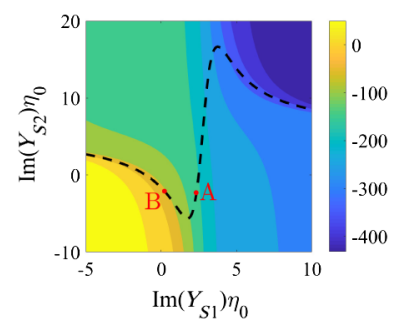

(b)
FIG. 16. (a) The transmittance $\left(\left|S_{12}\right|^{2}\right)$ and (b) the phase of the transmission coefficient performances of the QWP under given parameters $\left(\epsilon_{r}=3.55\right.$ and $\left.d=1.524 \mathrm{~mm}\right)$. Points $\mathrm{A}$ and $\mathrm{B}$ represent the idealized sheet admittance pairs of inner and outer sheets for $x$ and $y$ direction, respectively.

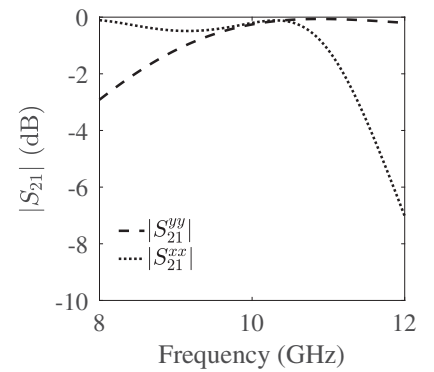

(a)

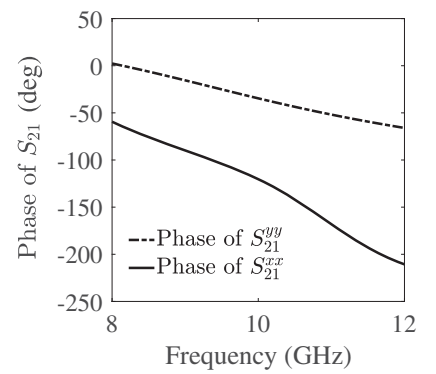

(b)
FIG. 17. On-axis simulation results of the designed QWP. (a) Amplitude and (b) phase of transmission coefficients for $x$ and $y$ polarizations.

for $x$ polarization: $\quad Y_{s 1}^{x x} \eta_{0}=2.325 j, \quad Y_{s 2}^{x x} \eta_{0}=-2.292 j$, $\angle S_{21}^{x x}=-134.3^{\circ}$; and point $\mathrm{B}$ for $y$ polarization: $Y_{s 1}^{y y} \eta_{0}=0.223 j, Y_{s 2}^{y y} \eta_{0}=-2.091 j, \angle S_{21}^{y y}=-43.28^{\circ}$. The metallic patterns of the inner and outer sheets were individually designed to realize these admittance values. The near-field coupling between the patterned metallic sheets was low and compensated for by slight adjustments to the inner and outer sheet patterns. The final unit cell dimensions are shown in Fig. 2(b).

The simulated results of the designed QWP for $x$ and $y$ polarizations are shown in Fig. 17. In simulation, the metallic cladding was modeled as zero-thickness copper with finite conductivity $\left(5.8 \times 10^{7} \mathrm{~S} / \mathrm{m}\right)$ boundary condition in the full-wave solver, ANSYS HFSS. At $10 \mathrm{GHz}$, the magnitudes of the transmission coefficients for $x$ and $y$ polarizations are -0.21 and $-0.26 \mathrm{~dB}$, respectively. The transmission phases for $x$ and $y$ polarizations are $-120^{\circ}$ and $-34^{\circ}$, respectively.

\section{APPENDIX B: EXTRACTED CIRCUIT MODEL OF THE BIREFRINGENT BOARD FOR THE PARALLEL POLARIZATION}

In this Appendix, a circuit model of the birefringent board for parallel polarization is demonstrated. The circuit model (see Fig. 18) was extracted from scattering
TABLE VIII. Detailed information and values of the extracted circuit parameters shown in Fig. 18.

\begin{tabular}{lc}
\hline \hline$Z_{0}$ & Extracted free space impedance, 301.6 $\Omega$ \\
$Z_{d}$ & Extracted substrate impedance, 203.34 $\Omega$ \\
$C_{g}$ & Extracted pattern capacitance, $0.1 \mathrm{pF}$ \\
$L_{1}$ & Extracted pattern inductance, $0.4 \mathrm{nH}$ \\
$L_{2}$ & Extracted pattern inductance, $1.84 \mathrm{nH}$ \\
$C_{2}$ & Extracted pattern capacitance, $0.675 \mathrm{pF}$ \\
$R$ & Extracted pattern resistance, $2400 \Omega$ \\
$C_{v}$ & Varactor diode capacitance \\
$d / 2$ & Substrate thickness, electrical length of $28.03^{\circ}$ \\
\hline \hline
\end{tabular}

simulations of the inner and outer sheets with the corresponding dielectric on each side. As shown in Fig. 6, the unit cell of the birefringent board has a width of $a=8 \mathrm{~mm}$ and a length of $b=10 \mathrm{~mm}$. Thus, the lump impedance of each sheet can be calculated by its scattering performance:

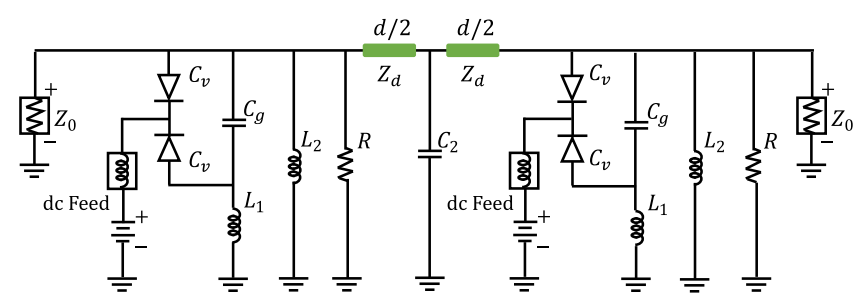

FIG. 18. Extracted circuit model of the designed birefringent board for the parallel polarization.

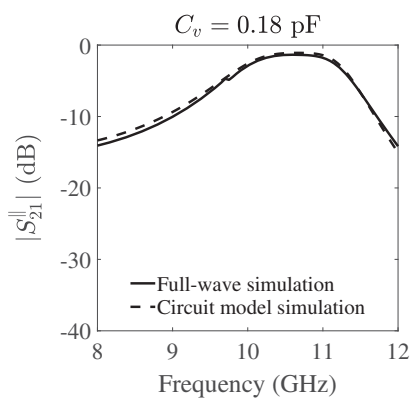

(a)

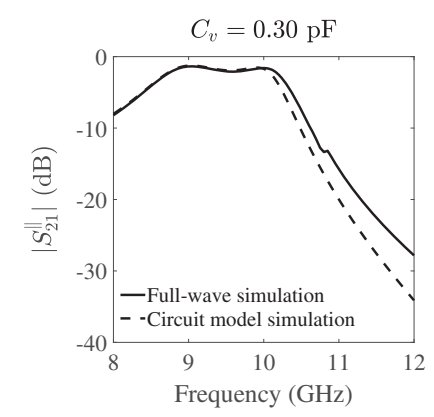

(c)

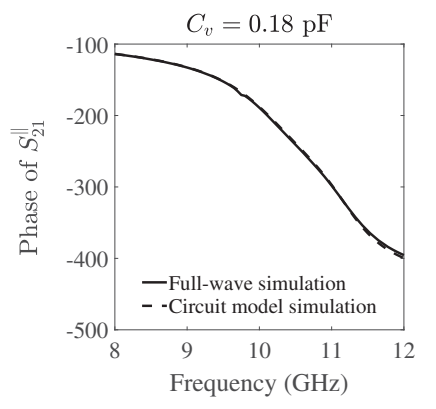

(b)

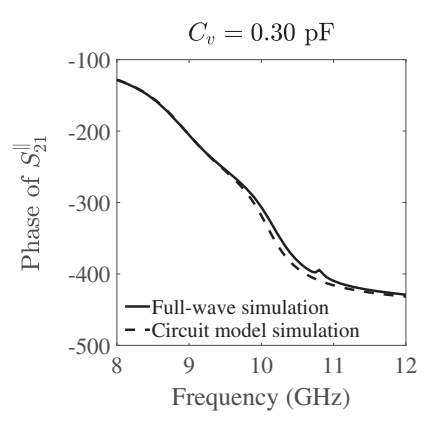

(d)
FIG. 19. Comparison between the full-wave simulation and the circuit model simulation of the birefringent board for the parallel polarization. 


$$
\begin{aligned}
Z^{\text {lump }} & =\frac{V}{I}=\frac{a}{b} \frac{E}{H} \\
& =0.8 \frac{E}{H} .
\end{aligned}
$$

The values of the circuit parameters extracted using Eq. (B1) are shown in Table VIII. The circuit shown in Fig. 18 was simulated with the commercial circuit solver Advanced Design System (ADS). The varactor diode $C_{v}$ was modeled using the SPICE model for MAVR-0001201411 varactors [31]. The simulation results of the circuit model in Fig. 18 are given for two representative varactor capacitances $\left(C_{v}=0.18 \mathrm{pF}\right.$ and $\left.C_{v}=0.30 \mathrm{pF}\right)$ in Fig. 19. The circuit simulations agree closely with full-wave simulations of the birefringent board. The close agreement confirms the accuracy of the circuit model shown in Fig. 5(c).

\section{APPENDIX C: MEASUREMENT RESULTS FOR BIREFRINGENT BOARDS UNDER DIFFERENT BIASING VOLTAGES}

The simulated and measured transmission coefficients of the birefringent boards for the parallel polarization are compared in this Appendix. For each bias voltage in the range $8-13 \mathrm{~V}$, the varactor capacitance is extracted by aligning the measured transmission phase to simulation. The extracted capacitances are shown in Table V. The simulated and measured transmission coefficients of Board $_{1}$ and Board 2 are shown in Fig 20. The varactor resistance is taken from Table I for each varactor capacitance used in simulation. Close agreement is observed between simulation and measurement, verifying the varactor diode parameters given in Table V.

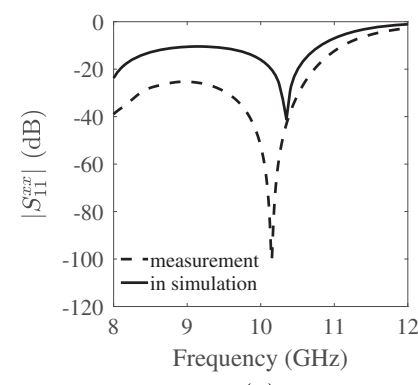

(a)

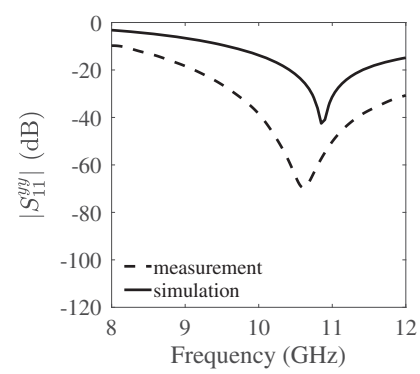

(c)

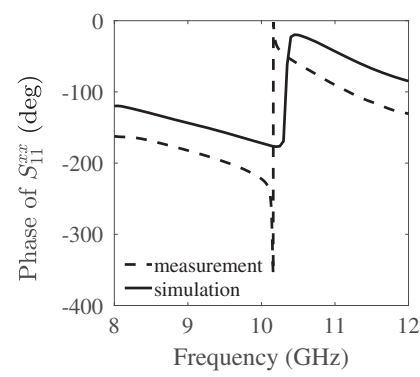

(b)

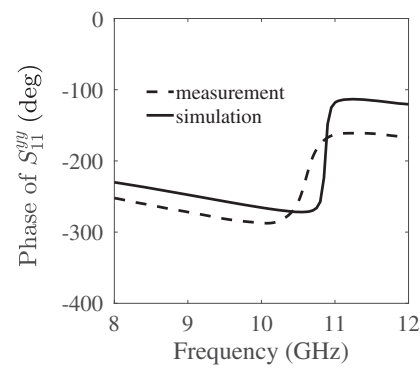

(d)
FIG. 21. Simulated and measured reflection coefficient entries for the QWP. (a) Amplitude and (b) phase of the reflection coefficients for $x$ polarization. (c) Amplitude and (d) phase of the reflection coefficients for $y$ polarization.

\section{APPENDIX D: MEASUREMENT RESULTS FOR BIREFRINGENT BOARDS UNDER DIFFERENT BIASING VOLTAGES}

The simulated and measured reflection coefficient entries for the QWPs and birefringent boards are shown here. The reflection coefficients for polarizations along the two orthogonal crystal axes of the QWP are shown in Fig. 21. The reflection coefficients remain below $-20 \mathrm{~dB}$ around the operating frequency.

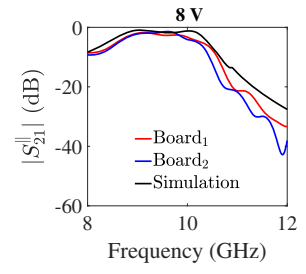

(a)

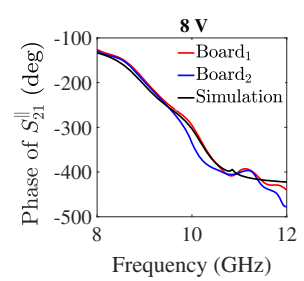

(g)

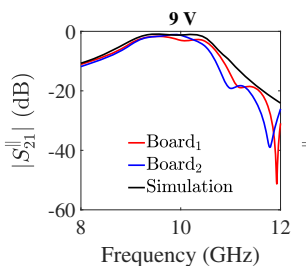

(b)

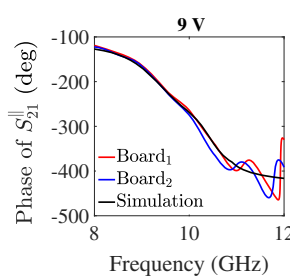

(h)

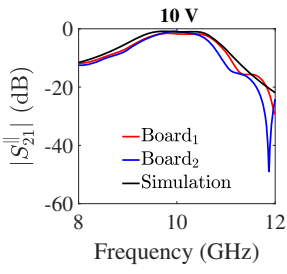

(c)

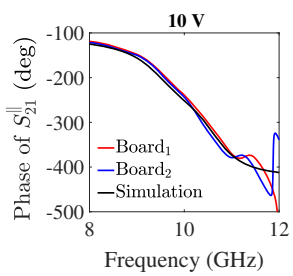

(i)

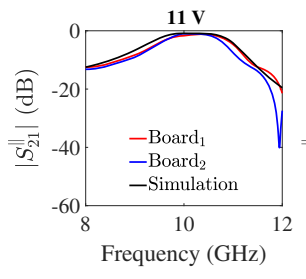

(d)

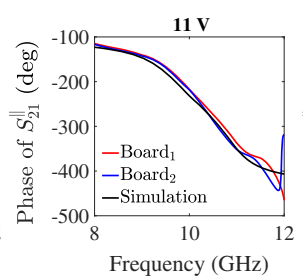

(j)

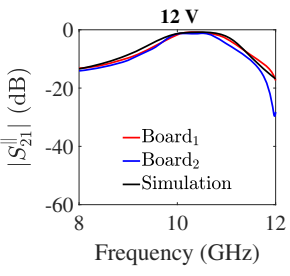

(e)

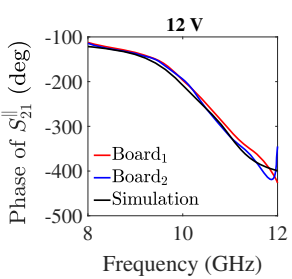

(k)

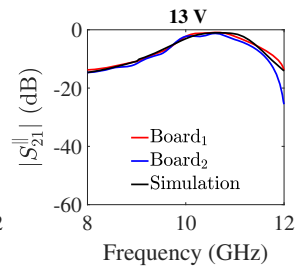

(f)

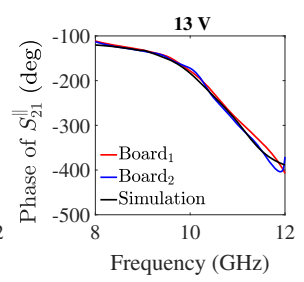

(l)

FIG. 20. Simulation and measurement results of the transmission coefficient for the parallel polarization of the birefringent boards, board $_{1}$ and board $_{2}$, for various biasing voltages. 


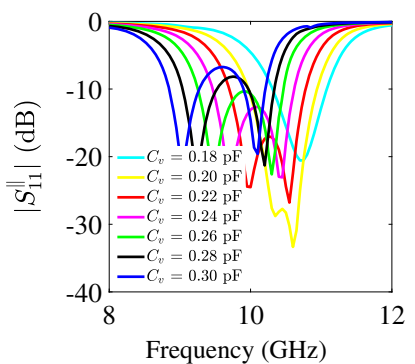

(a)

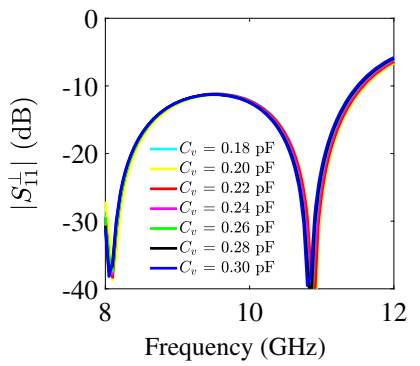

(c)

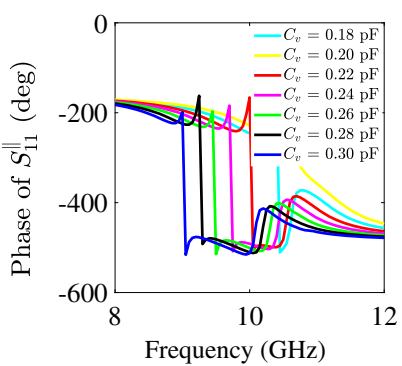

(b)

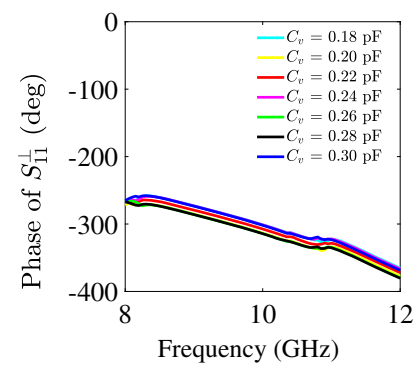

(d)
FIG. 22. Simulated reflection coefficient entries for the birefringent board. (a) Amplitude and (b) phase of the reflection coefficients for the parallel polarization. (c) Amplitude and (d) phase of the reflection coefficients for the perpendicular polarization.

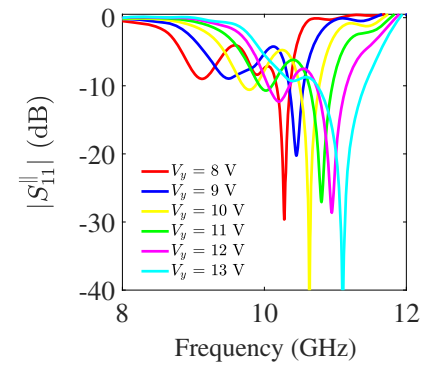

(a)

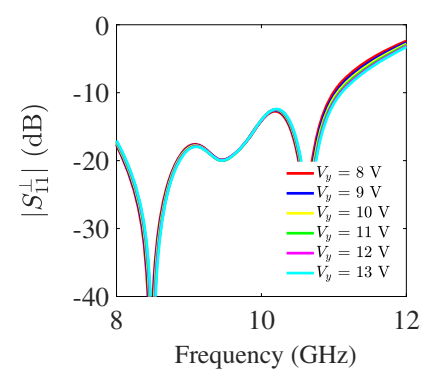

(c)

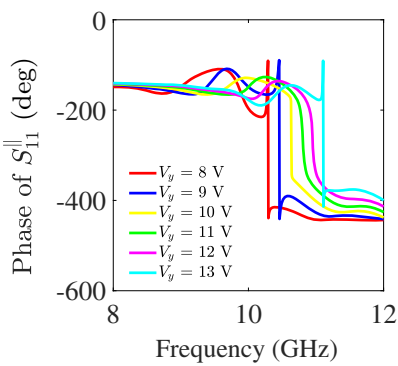

(b)

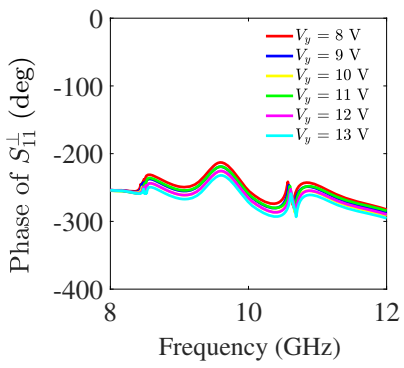

(d)
FIG. 23. Measured reflection coefficient entries for the birefringent board. (a) Amplitude and (b) phase of the reflection coefficients for the parallel polarization. (c) Amplitude and (d) phase of the reflection coefficients for the perpendicular polarization.
The simulated and measured reflection coefficients for the birefringent boards are shown in Figs. 22 and 23, respectively. For the parallel polarization, $\left|S_{11}\right|$ is not negligible. This results in reflections which reduce transmission and degrade the performances of the cascaded polarization rotator.

[1] C. L. Holloway, E. F. Kuester, J. A. Gordon, J. O’Hara, J. Booth, and D. R. Smith, An Overview of the Theory and Applications of Metasurfaces: The Two-Dimensional Equivalents of Metamaterials, IEEE Antennas Propag. Mag. 54, 10 (2012).

[2] A. V. Kildishev, A. Boltasseva, and V. M. Shalaev, Planar Photonics with Metasurfaces, Science 339, 1232009 (2013).

[3] C. Pfeiffer and A. Grbic, Metamaterial Huygens Surfaces: Tailoring Wave Fronts with Reflectionless Sheets, Phys. Rev. Lett. 110, 197401 (2013).

[4] N. Yu and F. Capasso, Flat Optics with Designer Metasurfaces, Nat. Mater. 13, 139 (2014).

[5] Y. Zhao, X.-X. Liu, and A. Alù, Recent Advances on Optical Metasurfaces, J. Opt. 16, 123001 (2014).

[6] S. A. Tretyakov, Metasurfaces for General Transformations of Electromagnetic Fields, Phil. Trans. R. Soc. A 373, 20140362 (2015).

[7] F. Zhang, L. Kang, Q. Zhao, J. Zhou, X. Zhao, and D. Lippens, Magnetically Tunable Left Handed Metamaterials by Liquid Crystal Orientation, Opt. Express 17, 4360 (2009).

[8] M. Decker, C. Kremers, A. Minovich, I. Staude, A. E. Miroshnichenko, D. Chigrin, D. N. Neshev, C. Jagadish, and Y. S. Kivshar, Electro-optical Switching by Liquid-Crystal Controlled Metasurfaces, Opt. Express 21, 8879 (2013).

[9] M. J. Dicken, K. Aydin, I. M. Pryce, L. A. Sweatlock, E. M. Boyd, S. Walavalkar, J. Ma, and H. A. Atwater, Frequency Tunable Near-Infrared Metamaterials Based on $\mathrm{VO}_{2}$ Phase Transition, Opt. Express 17, 18330 (2009).

[10] I. V. Shadrivov, A. B. Kozyrev, D. W. van der Weide, and Y. S. Kivshar, Tunable Transmission and Harmonic Generation in Nonlinear Metamaterials, Appl. Phys. Lett. 93, 161903 (2008).

[11] D. A. Smirnova, A. E. Miroshnichenko, Y. S. Kivshar, and A. B. Khanikaev, Tunable Nonlinear Graphene Metasurfaces, Phys. Rev. B 92, 161406 (2015).

[12] X. Miao, B. Passmore, A. Gin, W. Langston, S. Vangala, W. Goodhue, E. Shaner, and I. Brener, Doping Tunable Resonance: Toward Electrically Tunable Mid-Infrared Metamaterials, Appl. Phys. Lett. 96, 101111 (2010).

[13] H.-S. Ee and R. Agarwal, Tunable Metasurface and Flat Optical Zoom Lens on a Stretchable Substrate, Nano Lett. 16, 2818 (2016).

[14] P. Gutruf, C. Zou, W. Withayachumnankul, M. Bhaskaran, S. Sriram, and C. Fumeaux, Mechanically Tunable Dielectric Resonator Metasurfaces at Visible Frequencies, ACS Nano 10, 133 (2016).

[15] Z. Miao, Q. Wu, X. Li, Q. He, K. Ding, Z. An, Y. Zhang, and L. Zhou, Widely Tunable Terahertz Phase Modulation with Gate-Controlled Graphene Metasurfaces, Phys. Rev. X 5, 041027 (2015). 
[16] S. N. Burokur, J.-P. Daniel, P. Ratajczak, and A. De Lustrac, Tunable Bilayered Metasurface for Frequency Reconfigurable Directive Emissions, Appl. Phys. Lett. 97, 064101 (2010).

[17] M. C. Sherrott, P. W. C. Hon, K. T. Fountaine, J. C. Garcia, S. M. Ponti, V. W. Brar, L. A. Sweatlock, and H. A. Atwater, Experimental Demonstration of $>230^{\circ}$ Phase Modulation in Gate-Tunable Graphene-Gold Reconfigurable MidInfrared Metasurfaces, Nano Lett. 17, 3027 (2017).

[18] J. Zhao, Q. Cheng, J. Chen, M. Q. Qi, W. X. Jiang, and T. J. Cui, A Tunable Metamaterial Absorber Using Varactor Diodes, New J. Phys. 15, 043049 (2013).

[19] Y. Yao, R. Shankar, M. A. Kats, Y. Song, J. Kong, M. Loncar, and F. Capasso, Electrically Tunable Metasurface Perfect Absorbers for Ultrathin Mid-Infrared Optical Modulators, Nano Lett. 14, 6526 (2014).

[20] A. Fallahi and J. Perruisseau-Carrier, Design of Tunable Biperiodic Graphene Metasurfaces, Phys. Rev. B 86, 195408 (2012).

[21] J. Han, A. Lakhtakia, and C.-W. Qiu, Terahertz Metamaterials with Semiconductor Split-Ring Resonators for Magnetostatic Tunability, Opt. Express 16, 14390 (2008).

[22] E. Arbabi, A. Arbabi, S. M. Kamali, Y. Horie, M. FarajiDana, and A. Faraon, MEMS-Tunable Dielectric Metasurface Lens, Nat. Commun. 9, 812 (2018).

[23] D. F. Sievenpiper, J. H. Schaffner, H. J. Song, R. Y. Loo, and G. Tangonan, Two-Dimensional Beam Steering Using an Electrically Tunable Impedance Surface, IEEE Trans. Antennas Propag. 51, 2713 (2003).

[24] S. Liu, H.-X. Xu, H. C. Zhang, and T. J. Cui, Tunable Ultrathin Mantle Cloak via Varactor-Diode-Loaded Metasurface, Opt. Express 22, 13403 (2014).

[25] T. J. Cui, M. Q. Qi, X. Wan, J. Zhao, and Q. Cheng, Coding Metamaterials, Digital Metamaterials and Programmable Metamaterials, Light Sci. Appl. 3, e218 (2014).

[26] C. Pfeiffer and A. Grbic, Bianisotropic Metasurfaces for Optimal Polarization Control: Analysis and Synthesis, Phys. Rev. Applied 2, 044011 (2014).

[27] T. Niemi, A. O. Karilainen, and S. A. Tretyakov, Synthesis of Polarization Transformers, IEEE Trans. Antennas Propag. 61, 3102 (2013).
[28] Y. Ra'di and A. Grbic, Magnet-Free Nonreciprocal Bianisotropic Metasurfaces, Phys. Rev. B 94, 195432 (2016).

[29] J. A. Davis, D. E. McNamara, D. M. Cottrell, and T. Sonehara, Two-Dimensional Polarization Encoding with a Phase-Only Liquid-Crystal Spatial Light Modulator, Appl. Opt. 39, 1549 (2000).

[30] C. Pfeiffer and A. Grbic, Millimeter-Wave Transmitarrays for Wavefront and Polarization Control, IEEE Trans. Microwave Theory Tech. 61, 4407 (2013).

[31] MACOM Technology Solutions, solderable GaAs constant gamma flip-chip varactor diode, MAVR-000120-1411, available at https://www.macom.com/products/productdetail/MAVR-000120-14110P.

[32] D. M. Pozar, Microwave Engineering (John Wiley \& Sons, New York, 2009).

[33] M. A. Antoniades and G. V. Eleftheriades, Compact Linear Lead/Lag Metamaterial Phase Shifters for Broadband Applications, IEEE Antennas Wireless Propag. Lett. 2, 103 (2003).

[34] R. G. Meyer and M. L. Stephens, Distortion in VariableCapacitance Diodes, IEEE J. Solid-State Circuits 10, 47 (1975).

[35] S. M. Rudolph, C. Pfeiffer, and A. Grbic, Design and FreeSpace Measurements of Broadband, Low-Loss NegativePermeability and Negative-Index Media, IEEE Trans. Antennas Propag. 59, 2989 (2011).

[36] P. F. Goldsmith, Quasi-Optical Techniques, Proc. IEEE 80, 1729 (1992).

[37] S. Liu and T. J. Cui, Concepts, Working Principles, and Applications of Coding and Programmable Metamaterials, Adv. Opt. Mater. 5, 1700624 (2017).

[38] T. J. Cui, S. Liu, and L. Zhang, Information Metamaterials and Metasurfaces, J. Mater. Chem. C 5, 3644 (2017).

[39] X. G. Zhang, W. X. Jiang, H. W. Tian, and T. J. Cui, Controlling Radiation Beams by Low-Profile Planar Antenna Arrays with Coding Elements, ACS Omega 3, 10601 (2018).

[40] B. O. Raeker and A. Grbic, Compound Metaoptics for Amplitude and Phase Control of Wavefronts, arXiv: 1807.05461. 